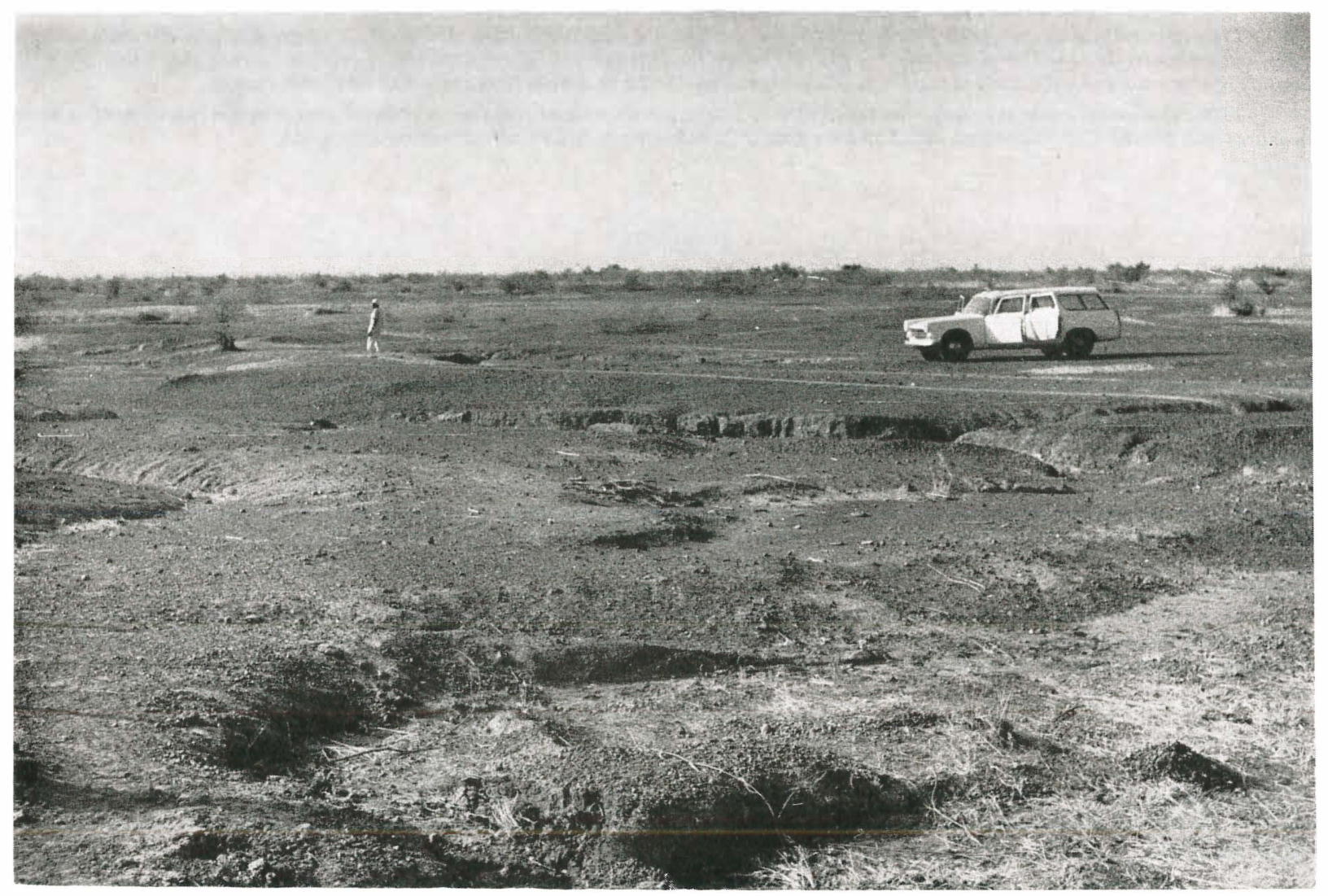

Photo Mariaux.

Erosion sur schistes. Boroya - Région de Ouahigouya.

\title{
RÉSULTATS \\ DE SIX ANS D'OBSERVATIONS SUR L'ÉROSION AU NIGER
}

\author{
par J. C. Delwaulle \\ Directeur du Centre Technique
}

Forestier Tropical du Niger, Haute-Volta

SUMMARY

\section{RESULTS OF SIX YEARS OF OBSERVATIONS OF EROSION IN NIGER}

The Centre Technique Forestier Tropical has set up a station in Niger to study erosion; the author reports on observations made over a period of six years. He deals successively with the environment, the station, the pluviometry, Wischmeier's $R$ factor characterizing the erosivity of the rainfall, run-off, erosion, the consequences of the nature of the soil and its working on erosion, and crop yield.

Revue Bois et Forêts des Tropiques, no 150, Juillet-Acût 1973 
The experimental set-up comprises 4 plots of land : a plot P1 with low contour-line walls ; a reference plot P2 without such walls, cultivated in the traditional manner ; a plot P3 where the vegetation is planted along the contour-lines ; and a plot P4 with mounds of stone covered with earth along the contour level. Plots 1, 3 and 4 were frequently harrowed and ridged.

This experiment made it possible in particular to determine the role of rainfall in erosion, and also the role of anti-erosion measures and manners of cultivation which prove effective in reducing erosion and increasing crop yield.

\section{RESUMEN}

\section{RESUltAdOS DE SEIS ANOS DE OBSERVACIONES ACERCA DE LA EROSION, EN EL NIGER}

El Centro Técnico Forestal Tropical, de Francia, ha instalado en el Niger una estación de estudio de la erosión y el autor da cuenta aqui de las observaciones llevadas a cabo durante seis años. Se procede sucesivamente al estudio del medio, de la estación de estudio, de la pluviometría, del factor $R$ de Wischmeier que caracteriza la erosividad de las lluvias, de la escorrentia, de la propia erosión, de las consecuencias del trabajo y del género del suelo sobre la erosión y, asimismo, el rendimiento de los cultivos.

El dispositivo experimental está formado por cuatro parcelas : una parcela P1, con muretes en curvas de nivel, una parcela testigo P2 sin acondicionamiento alguno y cultuvada según métodos tradicionales, una parcela P3 plantada en líneas de vegetación que se adaptan a las curvas de nivel y una parcela P4 con salientes formados por piedras recubiertas de tierra, también dispuestos en curvas de nivel. Las parcelas 1, 3 y 4 han sido labradas, habiéndose procedido a frecuentes labores de formación de caballones $y$ binazones.

En particular, esta experimentación ha permitido : determinar el papel desempeñado por la lluvia en la erosión, asi como el papel de los dispositivos antierosivos y las formas de cultivo que se manifiestan eficaces para disminuir la erosión y que permiten aumentar el rendimiento de los cultivos.

Une station d'étude de l'érosion a été installée par le Centre Technique Forestier Tropical au Niger en 1966 et cette station a été fonctionnelle jusqu'en 1971. La présente note fait la synthèse des principaux résultats obtenus au cours de ces six ans.

\section{I. - LE MILIEU}

Fig. 1

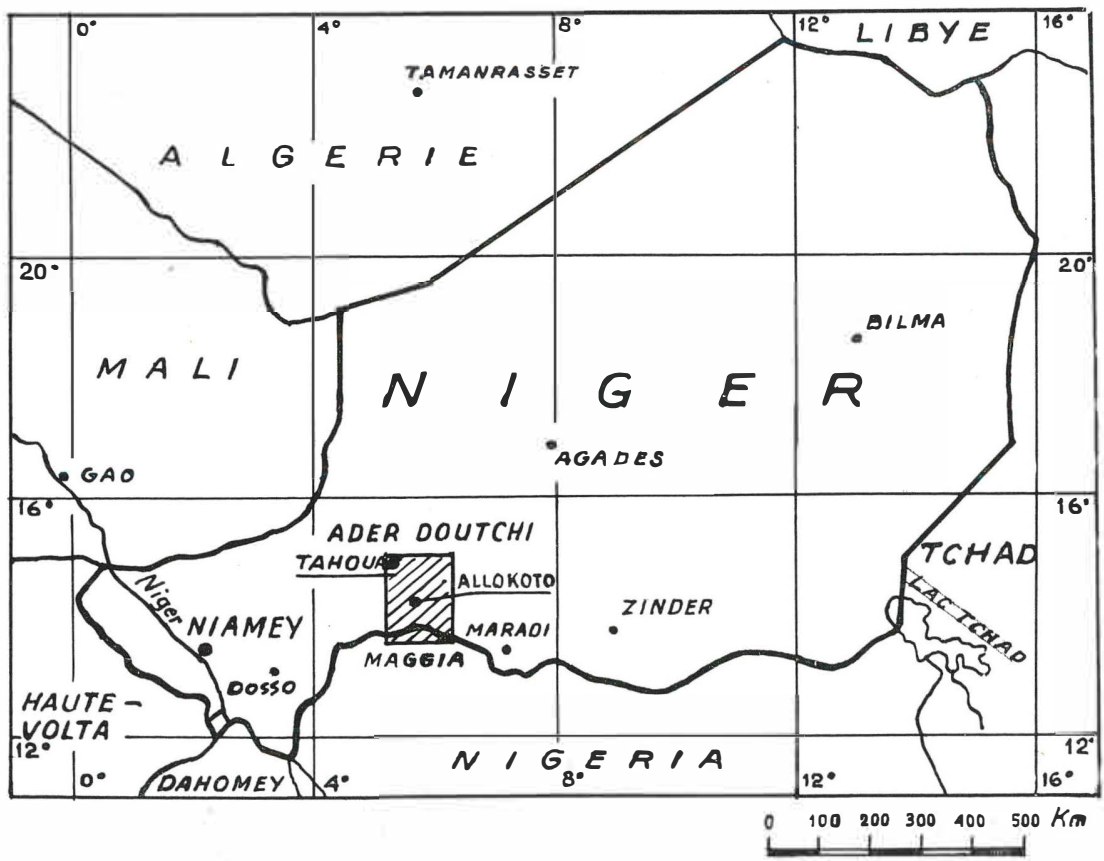

\section{1) SITUATION GÉNÉRALE.}

La station expérimentale d'étude de l'érosion a été installée à Allokoto (14\% $14^{\prime}$ lat. N, $5^{\circ} 38^{\prime}$ long. E).

Ce village est situé dans une petite vallée d'environ 18.000 hectares débouchant dans l'importante vallée de la Maggia, vallée située dans la région nommée Ader-DoutchiMaggia, relativement riche du point de vue agricole et qui fait l'objet depuis plus de dix ans d'actions importantes de développement agricole (voir carte de situation fig. 1).

Cette région est soumise à des phénomènes d'érosion particulièrement marqués et les travaux de D. R. S. (défense et restauration des sols) C. E. S. (Conservation des Eaux du Sol) nécessitaient 
l'étude des ruissellements, des pertes en terre et des dispositifs antiérosifs.

\section{2) LE CLIMAT}

La station météorologique la plus proche est située à Madaoua à $36 \mathrm{~km}$ à l'est d'Allokoto. La pluviométrie moyenne enregistrée de 1936 à 1970 (deux années manquantes 1938 et 1964) est de $495 \mathrm{~mm}$. La pluviométrie moyenne d'Allokoto doit être du même ordre, peut être légèrement inférieure du fait de la différence de latitude.

Nous avons dressé la courbe ombrothermique de cette station (fig. 2).

Le climat de Madaoua possède les caractéristiques suivantes :

Températures : Température moyenne annuelle $28^{\circ} 4$; minimum des températures moyennes mensuelles : $24^{\circ} 2$ en janvier; maximum des températures moyennes mensuelles : $33^{\circ}$ en mai.

La moyenne mensuelle des températures minimum ne descend pas au dessous de $15^{\circ} 8$ (janvier) et la moyenne mensuelle des températures maximum atteint $41^{\circ}$ en avril.

Pluviométrie : la pluviométrie totale est de $495 \mathrm{~mm}$ avec un maximum marqué en août $(197 \mathrm{~mm})$.

Répartition des pluies : On peut distinguer nettement deux saisons :

- une saison pluvieuse de quatre mois environ (juin à septembre) ; mai)

- une saison sèche de huit mois (octobre à

Hyǵrométrie, vent : En saison sèche, l'hygrométrie tombe à des valeurs extrêmes, parfois inférieures à $20 \%$. La dessiccation est encore aggravée de novembre à mars par l'harmattan, vent sec venant de l'Est.

Le déficit hydrique est donc considérable si l'on songe que la seule évaporation sur nappe libre dépasse $3 \mathrm{~m}$ par an.

\section{3) VÉGÉTATION.}

La végétation naturelle de la région est une savane arbustive constituée principalement de Combretum glutinosum, Combretum micranthum, Combretum nigricans, Guiera senegalensis, Acacia astringens, Acacia laeta, Piliostigma reticulatum, Anogeissus leiocarpus, Ziziphus mauritiaca, Poupartia birrea, Ficus gnaphalocarpus.

Cette végétation a subi fortement l'influence de l'homme et est actuellement très clairsemée. Sa composition ne doit refléter qu'imparfaitement ce que pouvait être la végétation climacique initiale.
Fig. 2

\section{MADAOUA}

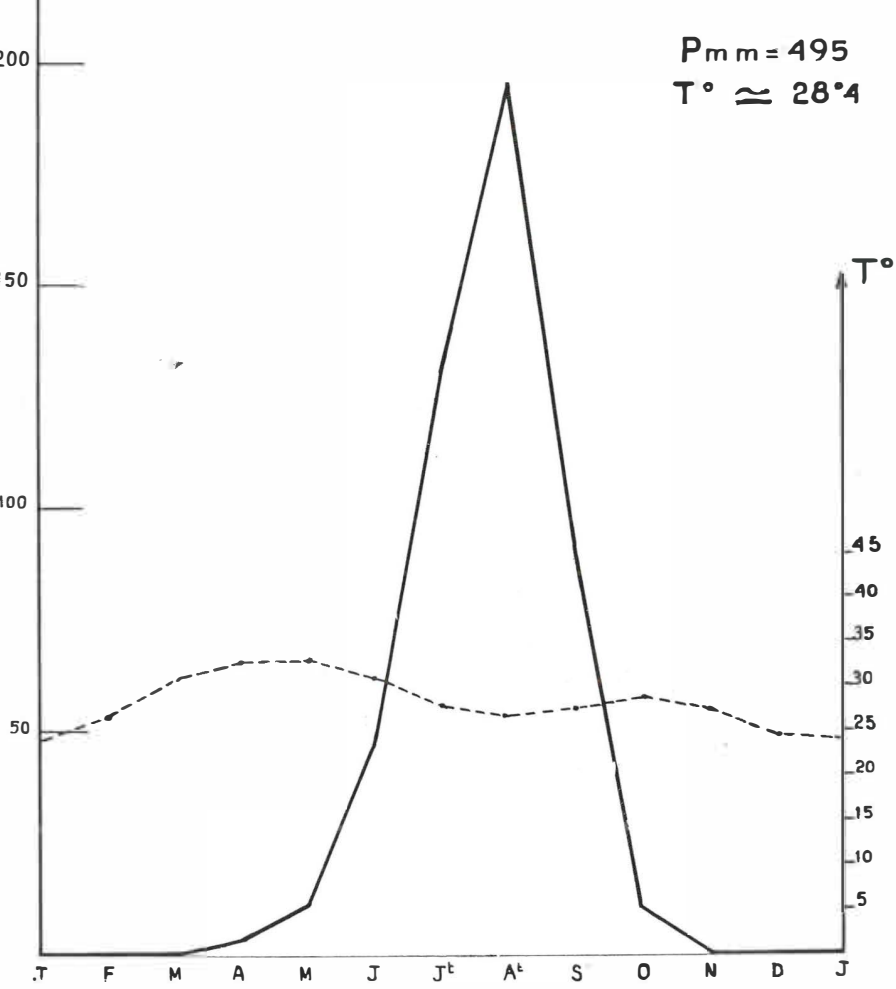

4) LE RELIEF ET LES SOLS.

La région se présente comme un plateau, largement entaillé par des vallées très larges, souvent inondables, le rebord du plateau est en corniche fréquemment et donne lieu à des pavages d'éboulis. Des versants et glacis d'épandage très amples raccordent ces corniches à la vallée (voir fig. 3).

La diversité des matériaux, grès, schistes, calcaires, apports dunaires etc. et des conditions de pédogénèse (topographie, action de l'eau, etc...), fait que les familles de sol sont en nombre très grand. Nous n'en donnerons pas le recensement, en signalant que la SOGETHA * a réalisé l'étude et la carte pédologique de la Maggia. Il faut insister sur le fait que ces sols sont parfois très riches et tout à fait exceptionnels dans ces régions d'Afrique, qu'il s'agisse de sols de vallée, ou de sols de versant ou de glacis, particulièrement lorsqu'ils ont pour origine les matériaux issus des schistes et calcaires de l'éocène qui donnent naissance à des rendzines, ou des sols bruns calcaires à tendance vertisolique.

* SOGETHA : Société générale des techniques hydroagricoles. 


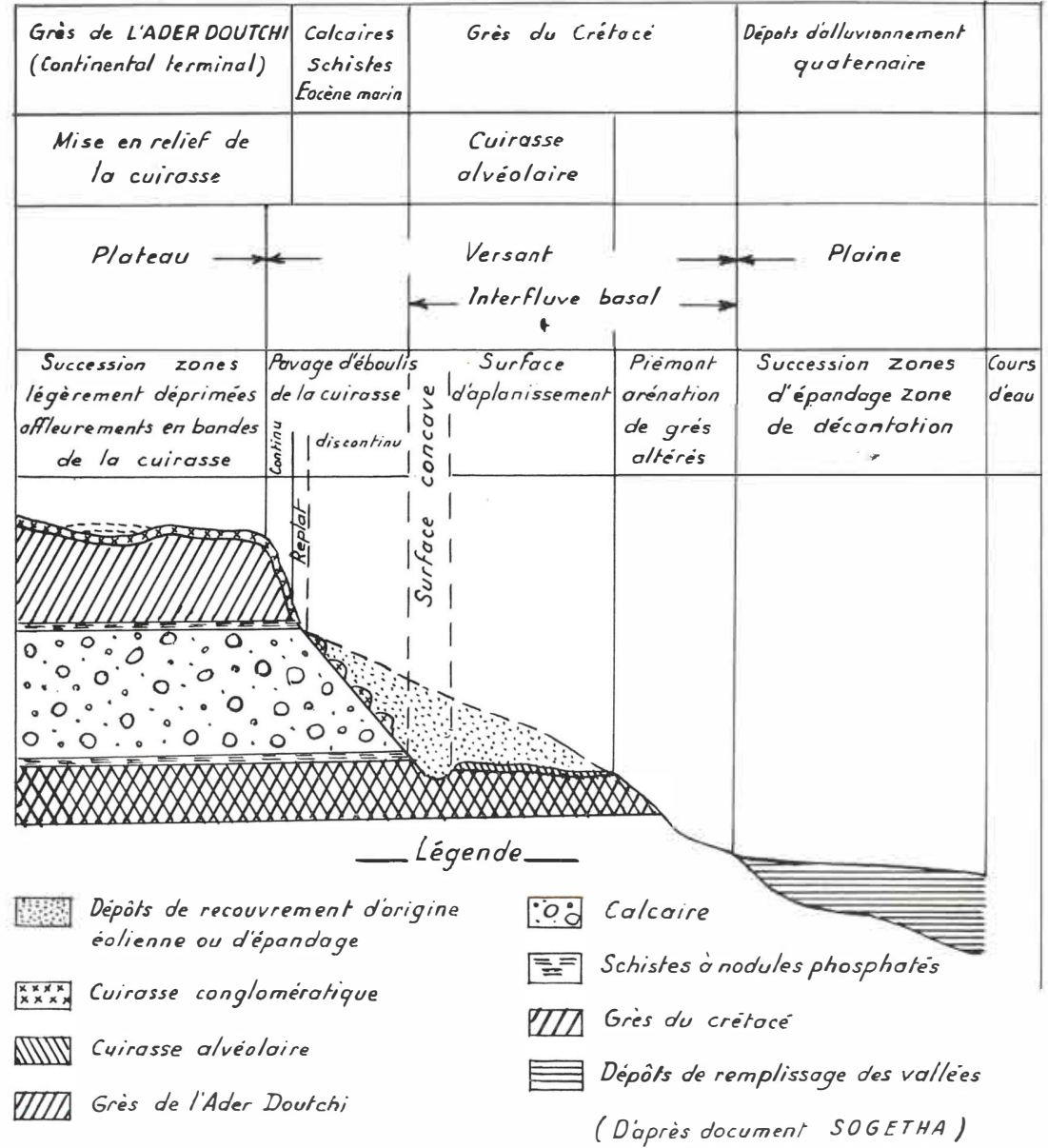

raides, des glacis dénudés, des surfaces cuirassées qui par leur très faible perméabilité provoquent des ruissellements intenses favorisant la dégradation des surfaces avoisinantes.

- Une érodibilité différentielle des formations géologiques.

- Un type de climat caractérisé par une saison des pluies à averses torrentielles.

- Des méthodes culturales sans souci de conservation du sol. L'action de l'homme et de ses animaux domestiques ont entraîné une dégradation générale de la couverture végétale.

La carte de Fournier place cette région dans les zones les plus menacées de l'Afrique, plus de $2.000 \mathrm{t} / \mathrm{km}^{2} / \mathrm{an} \mathrm{de}$ pertes en terre.

\section{6) LES HOMMES.}

La région est d'ethnie haoussa voisinant avec les Peuls et quelques Touaregs fixés. La densité de population est très forte dans les vallées. Les troupeaux sont très nombreux et très denses, : chèvres, moutons, ânes, bovins, chameaux. On voit couramment des troupeaux de chèvres de plusieurs centaines de têtes. L'action de l'homme et des animaux a provoqué dans certaines zones une "désertification" considérable particulièrement dangereuse.
Le complexe de la vallée de la Maggia est caractérisé par une érosion spectaculaire. En effet tous les facteurs favorisant cette érosion se trouvent réunis :

- Un relief marqué par des corniches très

\section{LA STATION}

\section{1) SITUATION ET CHOIX DE LA STATION.}

Le village d'Allokoto est situé dans une vallée affluente de la Haute-Maggia au Nord du village de Sabonga. Ses coordonnées sont les suivantes :

- 5038' longitude Est,

- $14^{\circ} 14$ latitude Nord,

- 365 m d'altitude.

Ce village fut choisi en partie en fonction du milieu humain. En effet les habitants avaient déjà le sens de la D. R. S. et avaient d'eux mêmes réalisé des cordons de pierre en courbe de niveau, de façon assez sommaire bien sûr. Il y avait là un aspect positif à ne pas négliger et qui pouvait être le ferment d'un aménagement ultérieur de D. R. S.

Le choix fut également déterminé par la nature des sols. On trouve, en effet, sur le territoire d'Allokoto des sols de versants très riches, sur matériaux issus de calcaires et schistes de l'éocène, sols de rendzine ou brun calcaire à tendance vertisolique. Ces terres sont classées par la SOGETHA comme terres à valeur agronomique exceptionnelle. Paradoxalement ces terres très riches étaient peu cultivées, et ne portaient que de pauvres cultures de sorgho. Ces sols sur pente de 2 à $5 \%$ sont menacés gravement par l’érosion, aussi faut-il nécessaire- 
ment utiliser les techniques de D. R. S. si on les met en culture.

On a pu trouver un terrain au nord d'Allokoto de superficie assez grande et de pente "identique " $3 \%$, représentatif de cette frange du versant, et susceptible de porter nos parcelles d'érosion. La végétation naturelle dans les parties non défrichées est composée d'Acacia seyal, Cymbopogon giganteum. La végétation spontanée après défrichement comprend essentiellement le Pennisetum pedicelletum, Schoenfeldia gracilis. Cette présence insolite de l'Acacia seyal sur ces pentes s'explique en fait par la fraîcheur permanente qui existe en profondeur.

\section{2) BUT DE L’ETUDE.}

Le but premier était de tester des aménagements antiérosifs associés à des façons culturales qui permettent de stopper le ruissellement et l'érosion, donc d'infiltrer une grosse partie des pluies. D'emblée nous avons choisi des méthodes simples, qui puissent être facilement mises en œuvre par des paysans encadrés (investissement humain), sans l'aide de moyens mécaniques (bouldozeur ou niveleuse).

D'autre part, dans un souci de diversification des cultures et de conservation du sol par les rotations culturales, il fallait démontrer qu'il était possible de cultiver autre chose que le sorgho, semé la plupart du temps sur ces terrains avec plus ou moins de succès ; l'arachide était traditionnellement réservée aux zones plus sableuses ou dunaires et le coton aux terres de vallée.
Nous avons donc réalisé des parcelles d'érosion sur lesquelles l'analyse des critères suivants a été effectuée en fonction du traitement antiérosif et du travail du sol.

- Intensité de la pluie.

- Intensité du ruissellement.

- Pertes en terres (dépôts solides et matière en suspension) analyse de ces pertes.

- Rendement des cultures.

La station a été créée en 1966 par Y. Birot et J. Galabert ; suivie en 1967 par Y. Birot, J. P. Thomassey, J. Galabert et J. P. Chapart ; en 1968 par Y. Birot, J. Galabert et J. P. Chapart ; de 1969 à 1971 par J. C. Delwaulle et B. Guez.

\section{3) LE DISPOSITIF EXPÉRIMENTAL.}

a) Les parcelles : Nous nous sommes éloignés volontairement de la méthode d'étude classique de l'érosion qui utilise des parcelles de faible dimension $\left(100\right.$ à $\left.500 \mathrm{~m}^{2}\right)$ et avons choisi des parcelles de tailles comparables à celles du champ du paysan haoussa.

Quatre parcelles constituent le dispositif, les trois premières ont été mises en place dès 1966, la quatrième en 1967 :

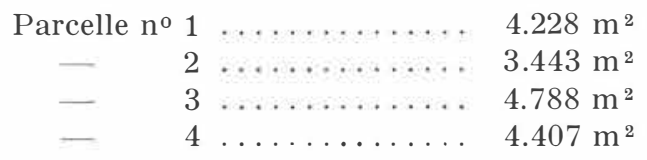

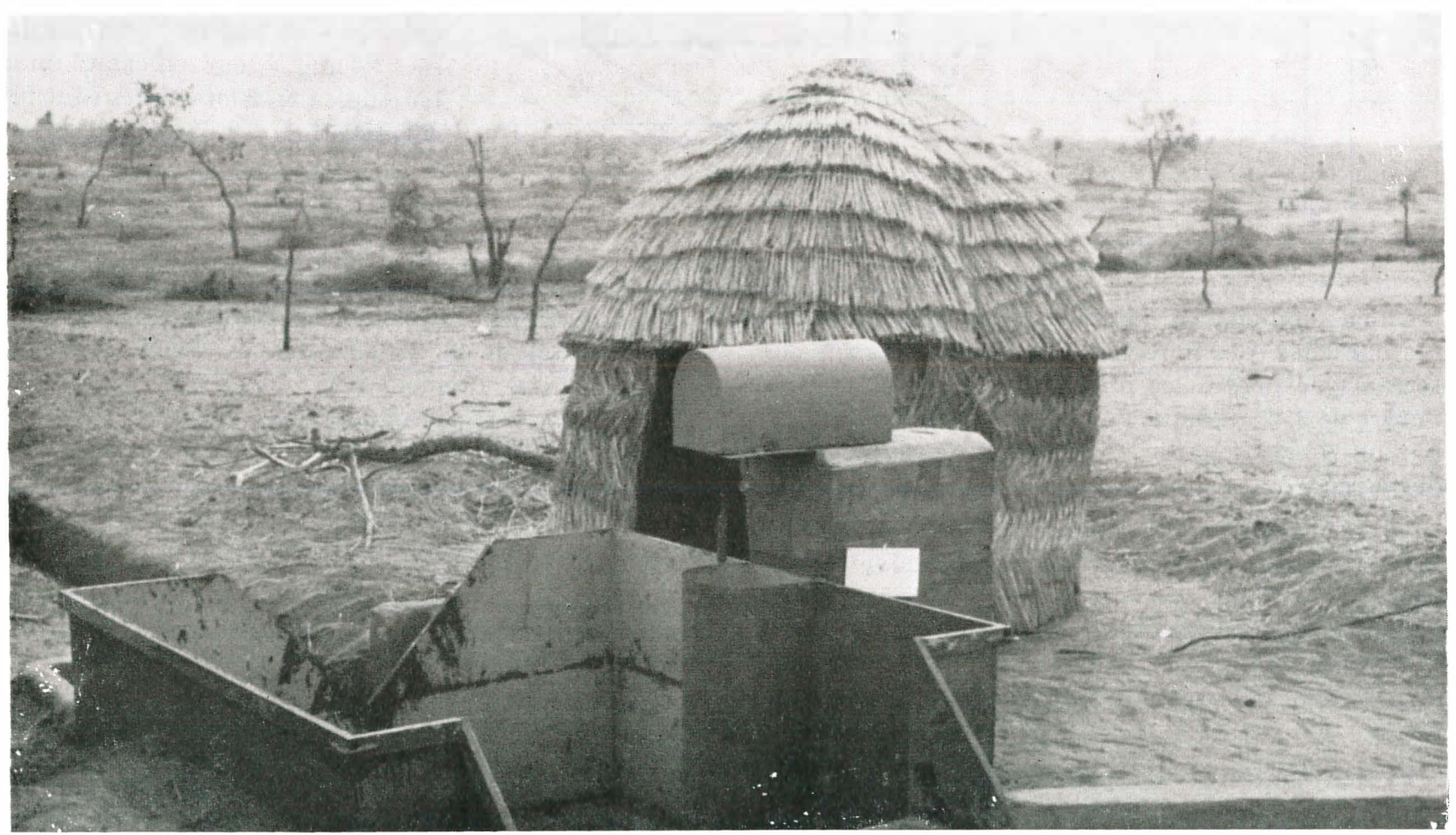




\section{$A \angle L O K O T O$}

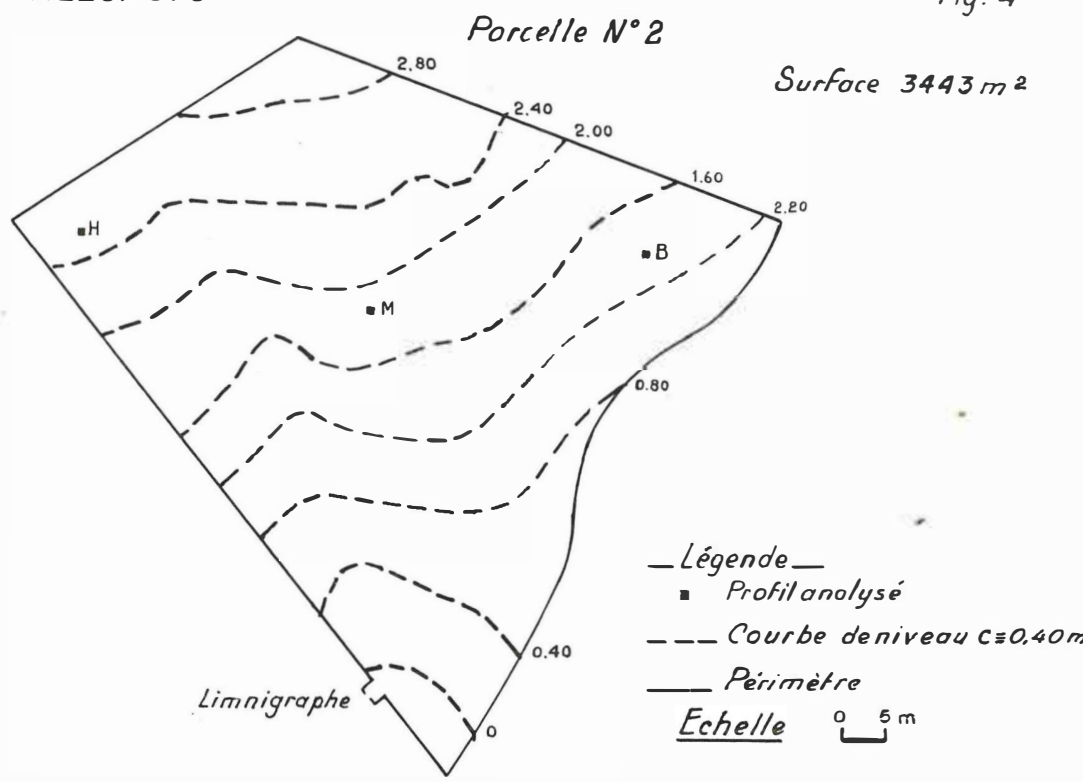

Cuve de ruissellement et déversoir.

Fig. 5

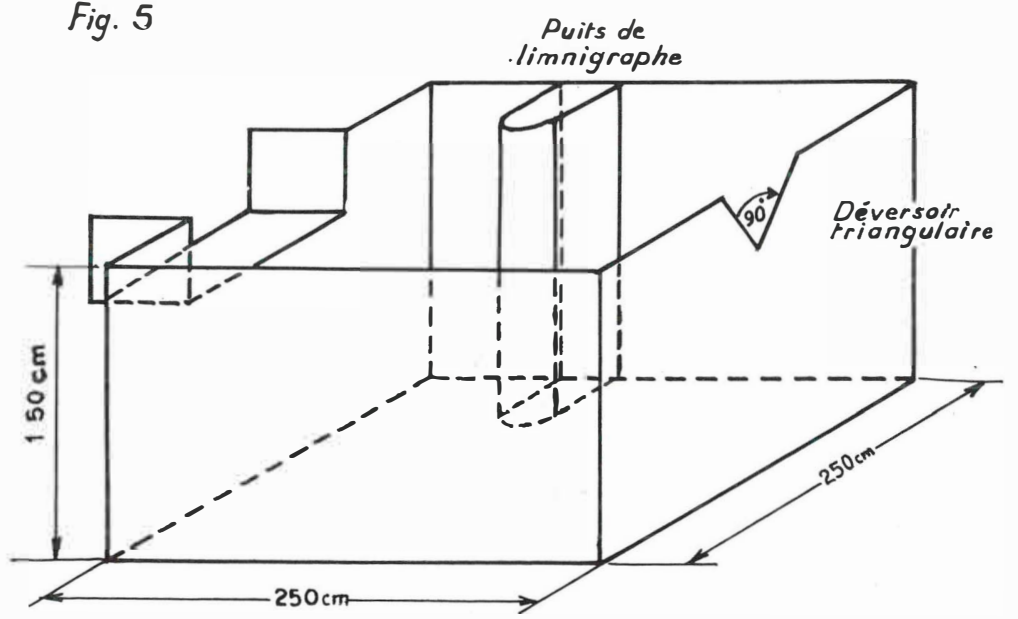

association et deux pluviographes à augets basculants. Ces derniers sont utilisés en particulier pour connaître l'intensité des pluies et calculer le facteur $R$ de l'équation de Wischimeier.

d) Fonctionnement de la station au cours d'une pluie : A u cours d'une pluie, les eaux de ruissellement, les éléments solides entraînés et ceux en suspension parviennent à la cuve où se déposent les éléments grossiers et une faible partie des éléments en suspension. Une part importante de l'eau, chargée de suspension est éliminée par le déversoir en $V$ et la quantité d'eau ainsi exportée est aisément calculée à l'aide du limnigramme.

Dès le début de l'écoulement du déversoir et pendant toute la durée de celui-ci, un opérateur prend à intervalles fixes des échantillons d'un litre qui serviront ultérieurement à procéder à l'évaluation des pertes de terre en suspension.

e) Les traitements étudiés : $\mathrm{Au}$ cours des six ans d'étude (5 pour la parcelle $n^{0} 4$ ). les traitements antiérosifs n'ont pas varié. Ils ont été implantés une fois pour toutes et nous n'avons procédé, au fil des ans, qu'à un entretien sommaire afìn d'étudier au mieux leur efficacité dans le temps. Ces traitements sont les suivants :

Parcelle 1: Des murets de pierre isohypses de 30 à $40 \mathrm{~cm}$ de haut ont été implantés en utilisant la formule de Ramser.

Nous donnons en fìgure 4 le croquis d'une de ces parcelles. Ces parcelles ont été choisies sur des terrains de pentes les plus homogènes possible de l'ordre de $3 \%$. Chaque parcelle est isolée de l'amont par un fossé de garde et est entourée d'une levée de terre.

b) Le système collecteur : Au point le plus bas de chaque parcelle est placée une cuve métallique (en ciment pour la parcelle 4) munie d'un déversoir triangulaire $\left(90^{\circ}\right)$ (fig. 5).

Chaque cuve est équipée d'un limnigraphe (échelle de réduction $1 / 25$, durée de révolution $12 \mathrm{~h})$.

c) Pluviomètres et pluviographes : Sur la station sont de plus installés deux pluviomètres
Cette formule donne la dénivelée des ouvrages en fonction de la pente : $\mathrm{H}=0,305(2+P / 4)$ ce qui nous donne, dans notre cas particulier $\mathrm{H} \sim 0,80 \mathrm{~m}$. La parcelle a été labourée ; cependant, en première année, du fait de manque de charrue, on a atteint un résultat proche du labour en effectuant un piochage. Des binages et billonnages fréquents ont été effectués.

PARCELle 2 : La parcelle témoin, n'a reçu aucun aménagement. Elle a été cultivée à la façon traditionnelle des Haoussas.

Parcelle 3 : La parcelle a été plantée en lignes de végétation isohypses (équidistance 2 fois plus faible que sur la parcelle 1). Le végétal utilisé est Andropogon gayanus introduit sur 3 lignes 
parallèles et en éclats de souche. Malheureusement, les conditions particulièrement défavorables du début d'hivernage 66 n'ont pas permis l'introduction correcte de ces lignes de végétation. Elles ont donc joué imparfaitement leur rôle. Les façons culturales ont été prévues identiques à la parcelle $n^{0} 1$ labour initial $\mathrm{y}$ compris.

Parcelle 4 : Cette parcelle n'a été implantée qu'en 1967. Le traitement antiérosif consiste en des bourrelets armés disposés à une équidistance identique aux murets du dispositif de la parcelle' I $(0,80 \mathrm{~m}$ de dénivelée). Ces bourrelets sont constitués d'un cordon isohypse de pierres provenant de l'épierrage de la parcelle, recouverts de terre. Ils ont environ $25 \mathrm{~cm}$ de haut. Ils ont été semés en graminées mais se sont surtout enherbés naturellement. Les façons culturales effectuées sur cette parcelle sont identiques à celles effectuées sur les parcelles 1 et 3 .

\section{III. - LA PLUVIOMÉTRIE}

Le tableau 1 donne les valeurs mensuelles de’ la pluviométrie enregistrée à Allokoto au cours des six ans d'observations et la figure 6 compare les courbes ombriques correspondantes, à la courbe de Madaoua établie sur les valeurs moyennes de 33 ans d'observations. On constate que les années 1967, 1969 et 170 accusent une répartition et une valeur totale proches de la moyenne.

L'année 1966 malgré un total (487) proche de la moyenne (495) présente une irrégularité dans la
Fig. 6
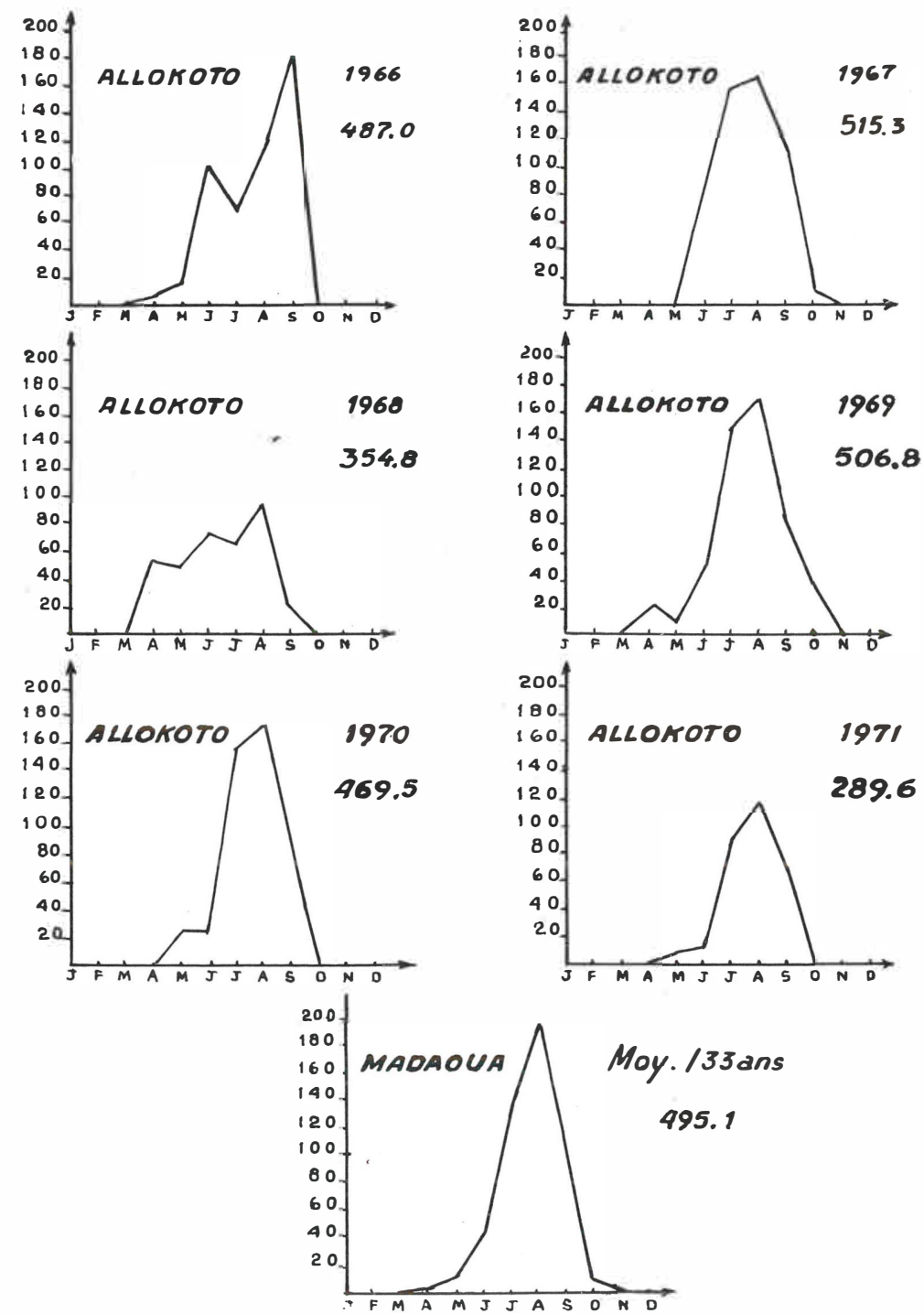

répartition surtout marquée par un déficit notable de pluviométrie en juillet : 70,9 contre 132,2.

TABLEAU 1

Station d'Allokoto

Pluviométrie

\begin{tabular}{|c|c|c|c|c|c|c|c|c|c|c|c|c|c|}
\hline & $\mathrm{J}$ & F & M & A & M & $\mathrm{J}$ & $\mathrm{Jt}$ & At & S & O & $\mathrm{N}$ & D & $\mathrm{T}$ \\
\hline $\begin{array}{l}1966 \ldots \\
1967 \ldots \\
1968 \ldots \\
1969 \ldots \\
1970 \ldots \\
1971 \ldots\end{array}$ & & & & $\begin{array}{r}5,3 \\
53,8 \\
20,1\end{array}$ & $\begin{array}{r}14,0 \\
47,2 \\
6,2 \\
24,5 \\
7,2\end{array}$ & $\begin{array}{r}101,0 \\
77,2 \\
73,2 \\
46,0 \\
22,0 \\
10,7\end{array}$ & $\begin{array}{r}70,9 \\
156,1 \\
64,8 \\
150,4 \\
158,4 \\
87,5\end{array}$ & $\begin{array}{r}113,3 \\
161,8 \\
95,4 \\
170,8 \\
172,7 \\
117,8\end{array}$ & $\begin{array}{r}179,8 \\
112,4 \\
19,9 \\
80,0 \\
91,9 \\
66,4\end{array}$ & $\begin{array}{r}2,7 \\
7,8 \\
33,3\end{array}$ & & & $\begin{array}{l}487,0 \\
515,3 \\
354,3 \\
506,8 \\
469,5 \\
289,6\end{array}$ \\
\hline $\begin{array}{l}\text { Moyenne } \\
\text { sur } 6 \text { ans }\end{array}$ & & & & 13,2 & 16,5 & 55,0 & 114,7 & 138,6 & 91,7 & 7,3 & & & 437,0 \\
\hline
\end{tabular}


TABLEAL 2

Station d'Allokoto

Nombre jours de pluie

\begin{tabular}{|c|c|c|c|c|c|c|c|c|c|c|c|c|c|}
\hline & $\mathbf{J}$ & $\mathrm{F}$ & M & A & M & $\mathrm{J}$ & $\mathrm{Jt}$ & At & $\mathrm{S}$ & $\mathrm{O}$ & $\mathrm{N}$ & D & $\mathrm{T}$ \\
\hline $\begin{array}{l}1966 \ldots \\
1967 \ldots \\
1968 \ldots \\
1969 \ldots \\
1970 \ldots \\
1971 \ldots\end{array}$ & & & & $\begin{array}{l}2 \\
1\end{array}$ & $\begin{array}{l}3 \\
\\
2 \\
2 \\
3 \\
2\end{array}$ & $\begin{array}{r}8 \\
7 \\
12 \\
7 \\
5 \\
3\end{array}$ & $\begin{array}{r}10 \\
9 \\
7 \\
9 \\
10 \\
8\end{array}$ & $\begin{array}{r}11 \\
13 \\
9 \\
8 \\
11 \\
13\end{array}$ & $\begin{array}{r}11 \\
7 \\
4 \\
6 \\
9 \\
5\end{array}$ & $\begin{array}{l}1 \\
2\end{array}$ & & & $\begin{array}{l}45 \\
38 \\
36 \\
36 \\
38 \\
31\end{array}$ \\
\hline
\end{tabular}

TABLEAU 3

Station d' Allokoto

Répartition des pluies par clasşes de hauteur

\begin{tabular}{|c|c|c|c|c|c|c|c|c|c|}
\hline & $0-10$ & $10-20$ & $20-30$ & $30-40$ & $40-50$ & $50-60$ & $60-70$ & $70-80$ & $\mathrm{~T}$ \\
\hline $\begin{array}{l}1966 \ldots \ldots \\
1967 \ldots \ldots \\
1968 \ldots \ldots \\
1969 \hat{e} \ldots \ldots \\
1970 \ldots \ldots \\
1971 \ldots \ldots \ldots\end{array}$ & $\begin{array}{l}26 \\
21 \\
24 \\
13 \\
23 \\
18\end{array}$ & $\begin{array}{r}10 \\
11 \\
6 \\
15 \\
7 \\
11\end{array}$ & $\begin{array}{l}7 \\
5 \\
2 \\
4 \\
3 \\
2\end{array}$ & $\begin{array}{l}1 \\
2 \\
3 \\
4 \\
3\end{array}$ & $\begin{array}{l}1 \\
1 \\
1 \\
1\end{array}$ & 1 & 1 & & $\begin{array}{l}45 \\
41 \\
36 \\
36 \\
38 \\
31\end{array}$ \\
\hline
\end{tabular}

L'année 1968 et surtout l'année 1971 sont des années fortement déficitaires et il est probable que récoltes et érosion s'en sont ressenties.

Le tableau 2 donne le nombre de pluies tombées par mois et le tableau 3 répartit ces pluies par classes de hauteurs.

Les pluies de hauteur inférieure à $20 \mathrm{~mm}$ occasionnent généralement peu de dégâts. Nous pouvons donc déjà prévoir que 1968 et surtout 1971 seront des années marquées par une érosion réduite.

Le tableau 4 ordonne les pluies par classes d'intensité maximum. Nous appelons intensité maximum, l'intensité la plus forte enregistrée durant une période d'au moins cinq minutes au cours d'une pluie (1).

TABLEAU 4

Station d'Allokoto

Répartition des intensités pluviométriques maximales par catégories

\begin{tabular}{|r|r|r|r|r|r|r|}
\hline $\begin{array}{c}I_{\max } \\
\mathrm{mm} / \mathrm{h}\end{array}$ & 1966 & 1967 & 1968 & 1969 & 1970 & 1971 \\
\cline { 2 - 5 } $0-24$ & 25 & 17 & 21 & 13 & 14 & 11 \\
$25-49$ & 7 & 7 & 7 & 12 & 15 & 15 \\
$50-74$ & 6 & 3 & 5 & 7 & 3 & 4 \\
$75-99$ & 5 & 9 & 1 & 3 & 1 & 1 \\
$100-124$ & - & 4 & 2 & - & 3 & - \\
$125-149$ & 2 & -1 & - & - & -1 & - \\
$150-174$ & - & 1 & - & - & 1 & - \\
\hline
\end{tabular}

Si nous considérons uniquement les pluies ayant eu une forte pointe d'intensité (supérieure à $74 \mathrm{~mm}$ / h), nous en trouvons en :

(1) L’intensité de la p'uie est exprimée en millimètres par heure.

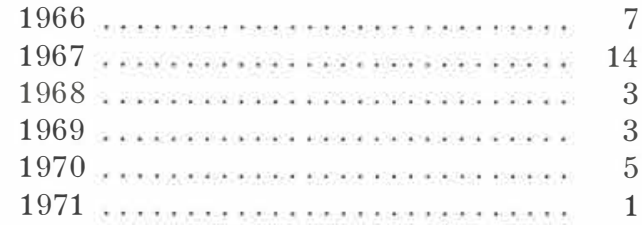

Ces pointes de forte intensité jouent un grand rôle dans l'érosion et nous pouvons supposer que 1967 sera l'année la plus érosive et 1971 la moins.

Le tableau 5 donne enfìn les valeurs annuelles de l'indice de Fournier. Cet indice d'agressivité climatique est le rapport du carré de la pluviométrie du mois le plus pluvieux $p$ à l'ensemble de la pluviométrie annuelle $P$.

TABLEAU 5

Station d'Allokoto

Indice de Fournier : $p^{2} / P$

\begin{tabular}{|r|r|r|r|r|r|r|}
\hline & 1966 & 1967 & 1968 & 1969 & 1970 & 1971 \\
& & & & & \\
\hline$P \ldots$ & 179,8 & 161,8 & 95,4 & 170,8 & 172,7 & 117,8 \\
$P$ & 487,0 & 515,3 & $\begin{aligned} 354,8 \\
25\end{aligned}$ & 506,8 & 469,5 & 289,6 \\
47,9 & 57,6 & 63,5 & 47,9 \\
$p^{2} \mid P$ & 66,4 & 50,8 & 25,6 & 57,6 & & \\
\hline
\end{tabular}

Valeur moyenne pour Madaoua établie sur la valeur moyenne de 33 ans (août : 197,1 mm)

$$
\frac{(197,1)^{2}}{495,1}=78,4 \text {. }
$$

A l'échelle annuelle, ce rapport a relativement peu de sens comme nous nous en rendrons compte ultérieurement en comparant cet indice aux valeurs du ruissellement et des pertes en terre. On peut se 
demander si on ne pourrait en améliorer l'intérêt en prenant comme période de référence non le mois officiel mais la période de trente jours $p_{30}$ durant laquelle il est tombé le maximum de pluies. Les valeurs de cet indice modifié sont données par le tableau 6 .

\section{TABLEAU 6}

Station d'Allokoto

Indice de Fournier modifié : $I=p_{30}^{2} / P$

\begin{tabular}{|c|c|c|c|c|c|c|}
\hline & 1966 & 1967 & 1968 & 1969 & 1970 & 1971 \\
\hline$p_{30} \ldots$ & 208,1 & 219,2 & 111,2 & 183,9 & 253,3 & 146,4 \\
\hline$P$ & 487,0 & 515,3 & 354,8 & 506,8 & 469,5 & 289,6 \\
\hline$p_{30}^{2} / P$ & 88,92 & 91,5 & 34,85 & 66,73 & 136,66 & 74,00 \\
\hline
\end{tabular}

\section{IV. - LE FACTEUR R}

Wischmeier a défini un facteur $R$ qui caractérise l'érosivité des pluies.

Rappelons ce qu'est exactement ce facteur :

C'est le produit de l'énergie globale Eg de la pluie par l'intensité maximum enregistrée durant 30 minutes $\left(\mathrm{I}_{30}\right)$, divisé par 100 .

$$
\mathrm{R}=\frac{\mathrm{Eg} \times \mathrm{I}_{30}}{100} .
$$

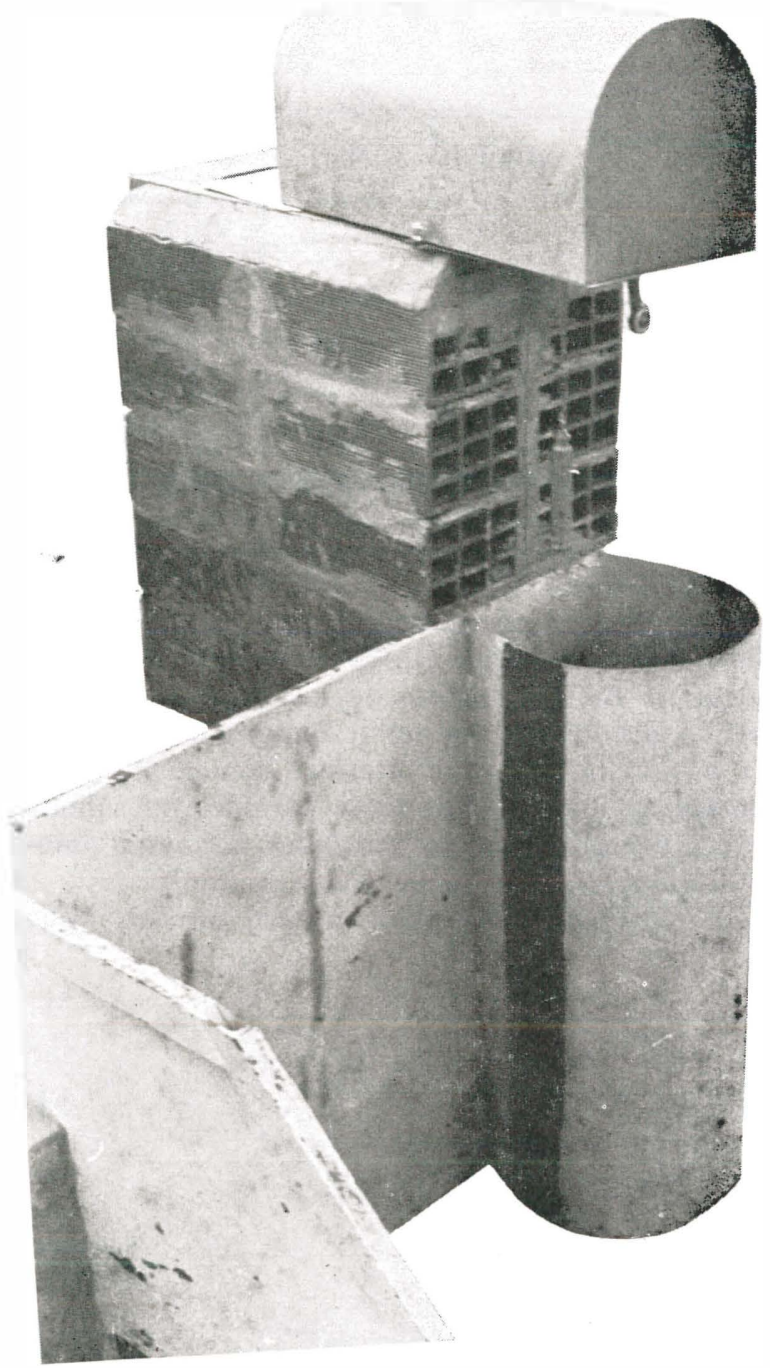

En passant aux unités métriques, $\mathrm{Eg}$ en $\mathrm{tm}$ par $\mathrm{Km}^{2}$ $(1 \mathrm{tm}=1000 \mathrm{Kgm})$ et $\mathrm{I}_{30}$ en $\mathrm{mm}$ par heure la formule devient :

$$
\begin{aligned}
\mathrm{R} & =\frac{1}{1.7 \overline{35,6}} \times \\
& \times \frac{\mathrm{Eg} \times \mathrm{I}_{30}}{100} .
\end{aligned}
$$

Eg est la somme des énergies unitaires Eu, l'énergie unitaire étant l'énergie pro-

Déversoir en " $V$ ". A droite, puits du limnigraphe.

Photo Delwaulle.

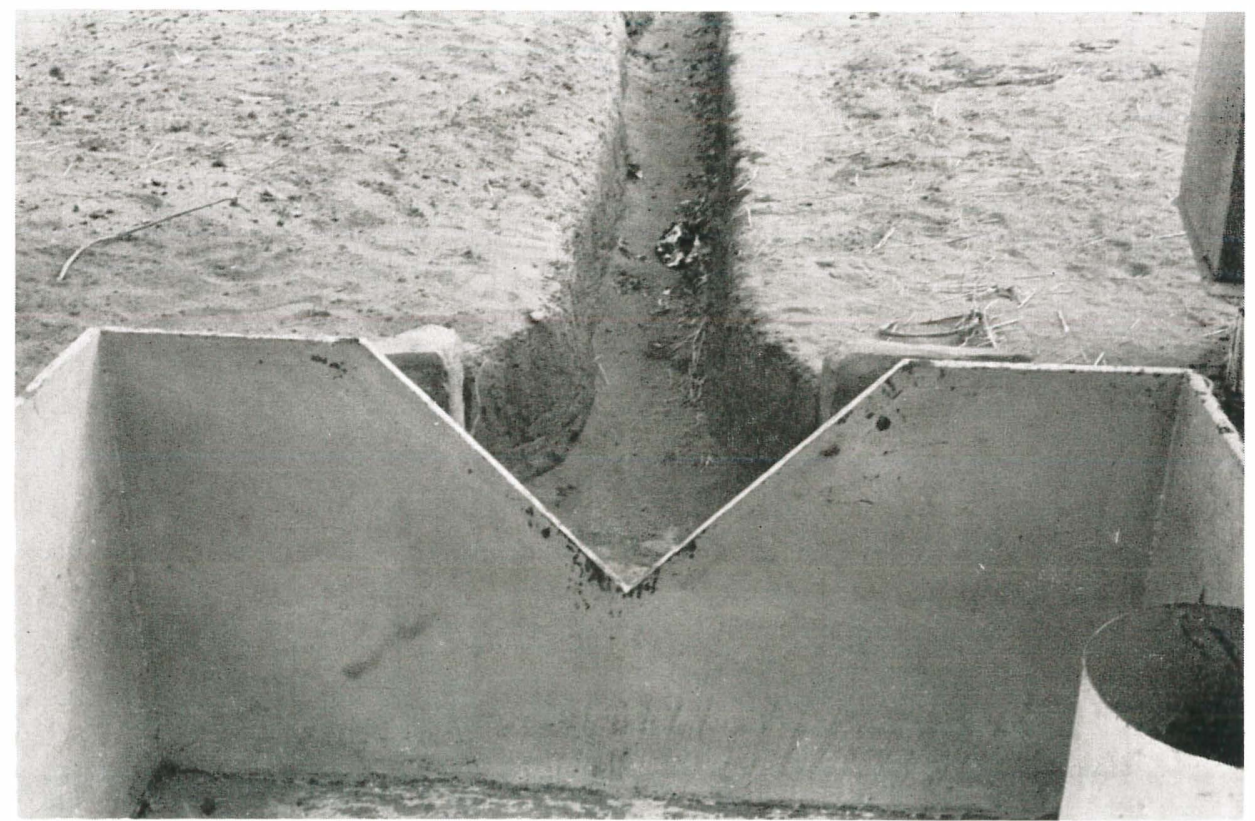


TABLEAU 7

Station d'Allokoto

L'indice $\mathrm{Rm}$ mensuel

\begin{tabular}{|c|c|c|c|c|c|c|c|c|}
\hline & Avril & Mai & Juin & Juillet & Août & Septembre & Octobre & Total \\
\hline 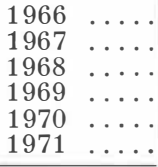 & $\begin{array}{r}29,20 \\
5,78\end{array}$ & $\begin{array}{r}28,92 \\
\\
5,06 \\
2,27\end{array}$ & $\begin{array}{r}54,30 \\
28,28 \\
9,75 \\
3,69 \\
9,02 \\
3,88\end{array}$ & $\begin{array}{r}28,21 \\
113,15 \\
17,22 \\
51,59 \\
83,74 \\
53,78\end{array}$ & $\begin{array}{r}23,55 \\
52,16 \\
65,48 \\
78,57 \\
117,96 \\
43,05\end{array}$ & $\begin{array}{r}74,29 \\
112,24 \\
\\
32,84 \\
35,10 \\
49,67\end{array}$ & 5,50 & $\begin{array}{l}180,35 \\
305,83 \\
150,57 \\
177,97 \\
250,88 \\
152,65\end{array}$ \\
\hline $\begin{array}{l}\text { Moyenne } \\
\text { sur } 6 \text { ans. . }\end{array}$ & 5,83 & 6,04 & 18,40 & 56,37 & 63,36 & 47,60 & 0,92 & 198,52 \\
\hline
\end{tabular}

duite par la chute de $1 \mathrm{~mm}$ de pluie, cette énergie est évidemment fonction de l'intensité de la pluie.

Pratiquement on découpe les pluies en périodes d'intensité homogène (Ih), une abaque donne la valeur de $\mathrm{Eu}$ en fonction de $\mathrm{Ih}(\mathrm{Eu}=a+b \log$ Ih). Le produit de Eu par le nombre de mm tombés durant la période d'intensité homogène donne l'énergie globale durant cette période. La somme de ces énergies globales partielles permet d'obtenir Eg d'où $R, \mathrm{I}_{30}$ étant exprimé en $\mathrm{mm} / \mathrm{h}$.

On calcule l'indice pluie moyen annuel, que
Wischmeier appelle aussi indice d'érosion, en analysant les relevés pluviométriques de toutes les pluies tombées au cours de l'année. Les Américains ne retiennent d'ailleurs que les pluies dont la hauteur est au moins égale à $1 / 2$ pouce $(12,7 \mathrm{~mm})$ et considèrent qu'une période de $6 \mathrm{~h}$ au cours de laquelle il est tombé moins de 0,05 pouce $(1,27 \mathrm{~mm})$ marque la séparation entre 2 pluies.

Nous avons calculé cet indice : Le tableau 7 donne les valeurs mensuelles.

Le calcul de la valeur de $R$ est relativement long
$\mathbf{R}$

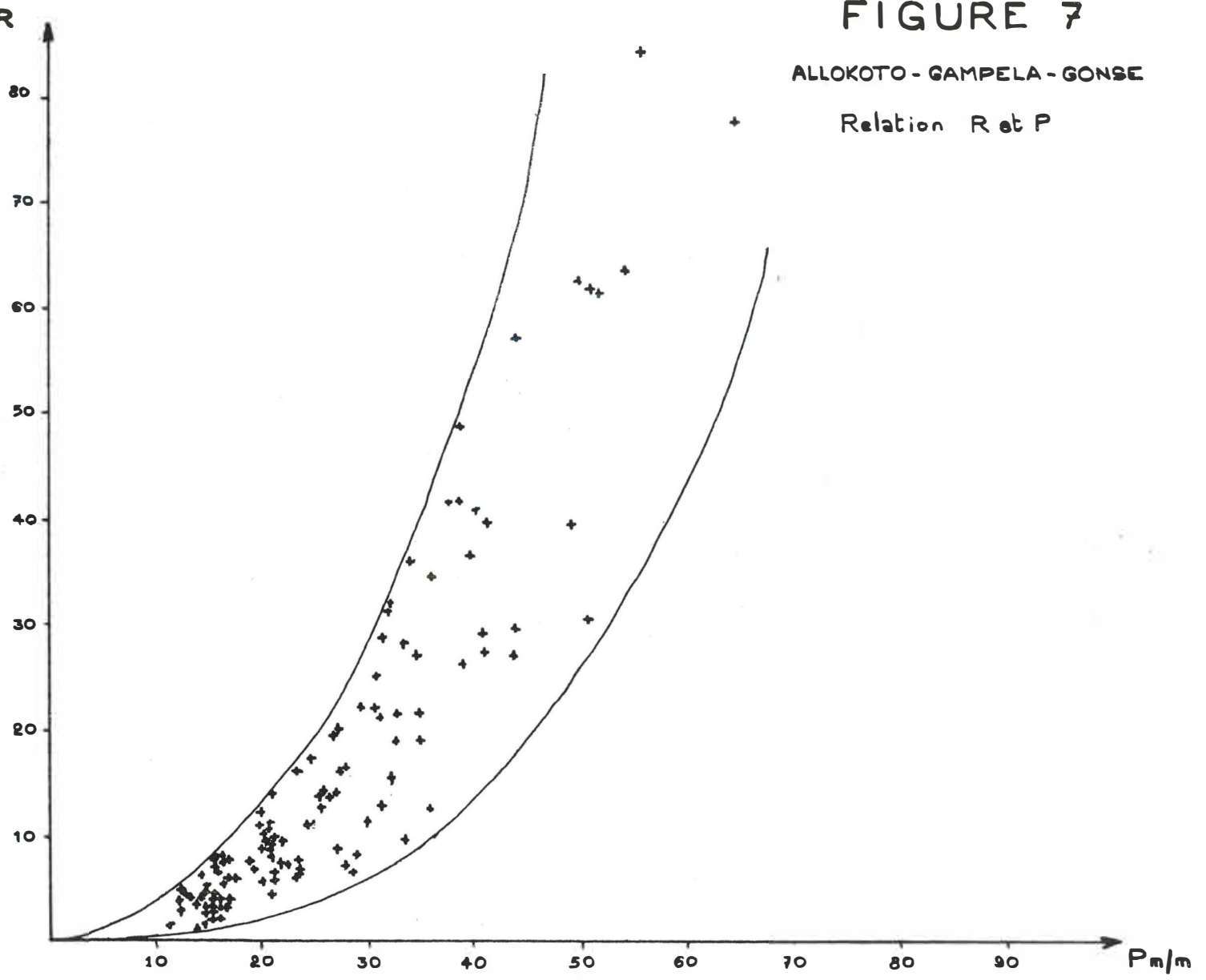


mais il caractérise réellement l'érosivité de chaque pluie et sa sommation sur l'année est un excellent indice caractérisant l'érosivité de l'année comme nous le verrons lors de l'étude du ruissellement et des pertes en terre.

Sa moyenne réalisée sur un grand nombre d'années permettrait d'établir une carte donnant ses valeurs moyennes, carte qui serait d'un grand intérêt en Défense et Restauration des Sols.

Il était donc tentant d'essayer de calculer rapidement $R$ en fonction de valeurs données par les stations météorologiques et la première idée était de voir si une relation satisfaisante n'existait pas entre $R$ et $P$.

La figure 7 donne les valeurs de $R$ en fonction de $P$ pour les années 67 à 69 d'Allokoto, 67 à 69 de Gampela et 69 de Gonsé. Gampela et Gonsé étant deux stations de Haute-Volta près de Ouagadougou où nous avons effectué des recherches en matière de Défense et Restauration des Sols. Comme on peut le constater, les valeurs de $R$ sont relativement concentrées pour de faibles valeurs de $P(10$ à $20 \mathrm{~mm}$ ) mais la dispersion est accusée pour les fortes valeurs : Pour $P$ voisin de $50 \mathrm{~mm}, R$ varie de 30 à 80 . On peut faire rentrer le nuage de points entre deux courbes paraboliques et la dispersion a une allure générale de "queue de comète".

Il n'y a donc pas de relation directe entre $R$ et $P$ et cela ne doit pas nous étonner puisque dans le calcul de $R$ nous faisons intervenir l'intensité maximale en 30 minutes ainsi que les intensités homogènes successives.

Il est alors tentant de faire intervenir le facteur $I_{30}$ facilement mensurable et nous avons essayé de voir s'il existait, pour les mêmes données que précédemment une relation entre $R$ et $P \times I_{30}$ (fìg. 8).

Le nuage de points s'oriente visiblement selon une droite et une relation du type $R=a P I_{30}+b$ semble pouvoir être défìnie. Le coefficient de corrélation est très satisfaisant (supérieur à 0,98 ) et l'éqúation finale s'écrit

$$
R=0,01572 P I_{30}-1,179 .
$$

Une relation aussi étroite montre que $P$ et $I_{30}$ suffìsent à caractériser une pluie ce qui signifie que les pluies sont de types voisins et que les régimes d'Allokoto au Niger et Gampela-Gonsé en Haute-Volta présentent de grandes similitudes.

L'équation peut donc être valablement utilisée pour le calcul de $R$ pour les stations de la zone. Il

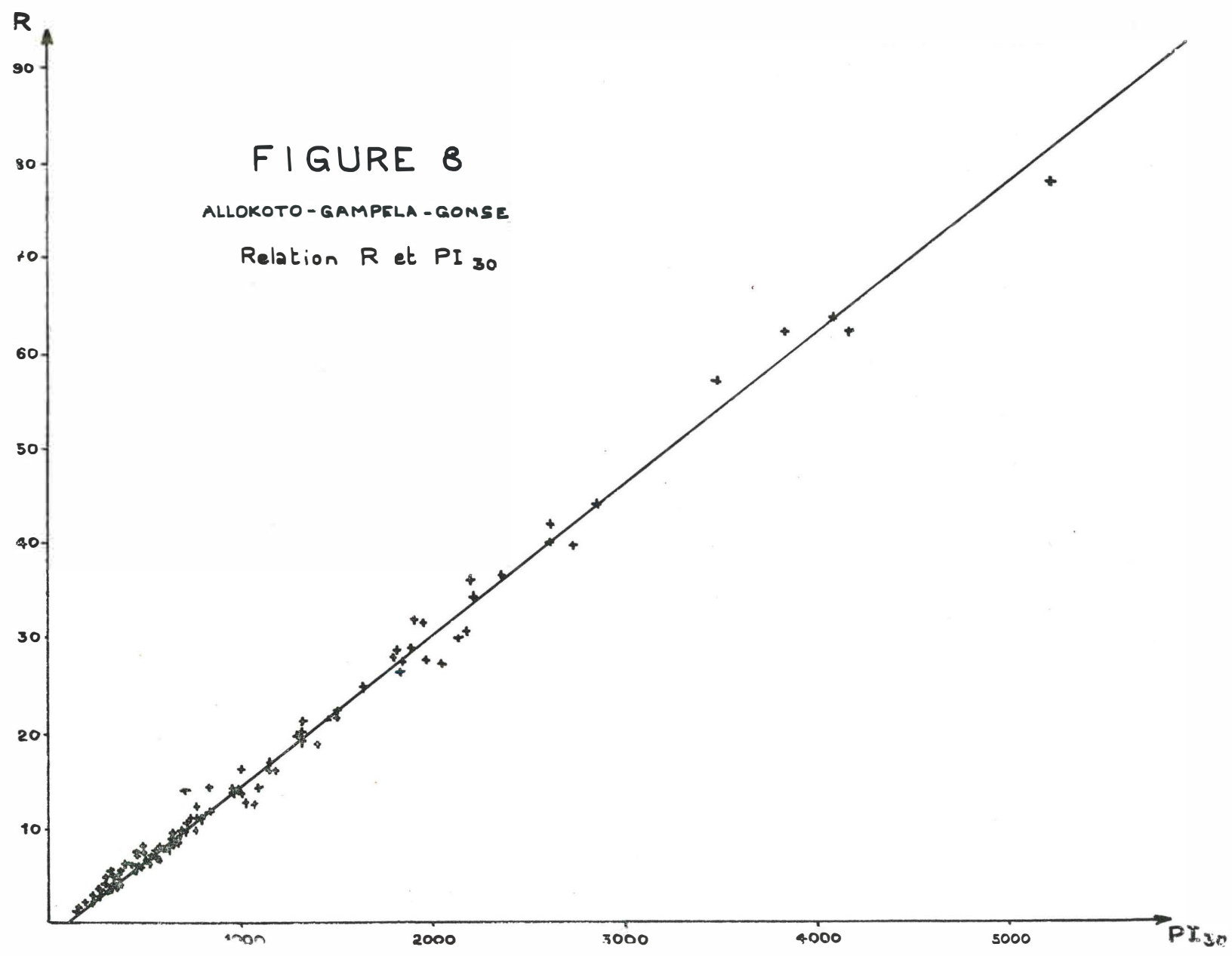




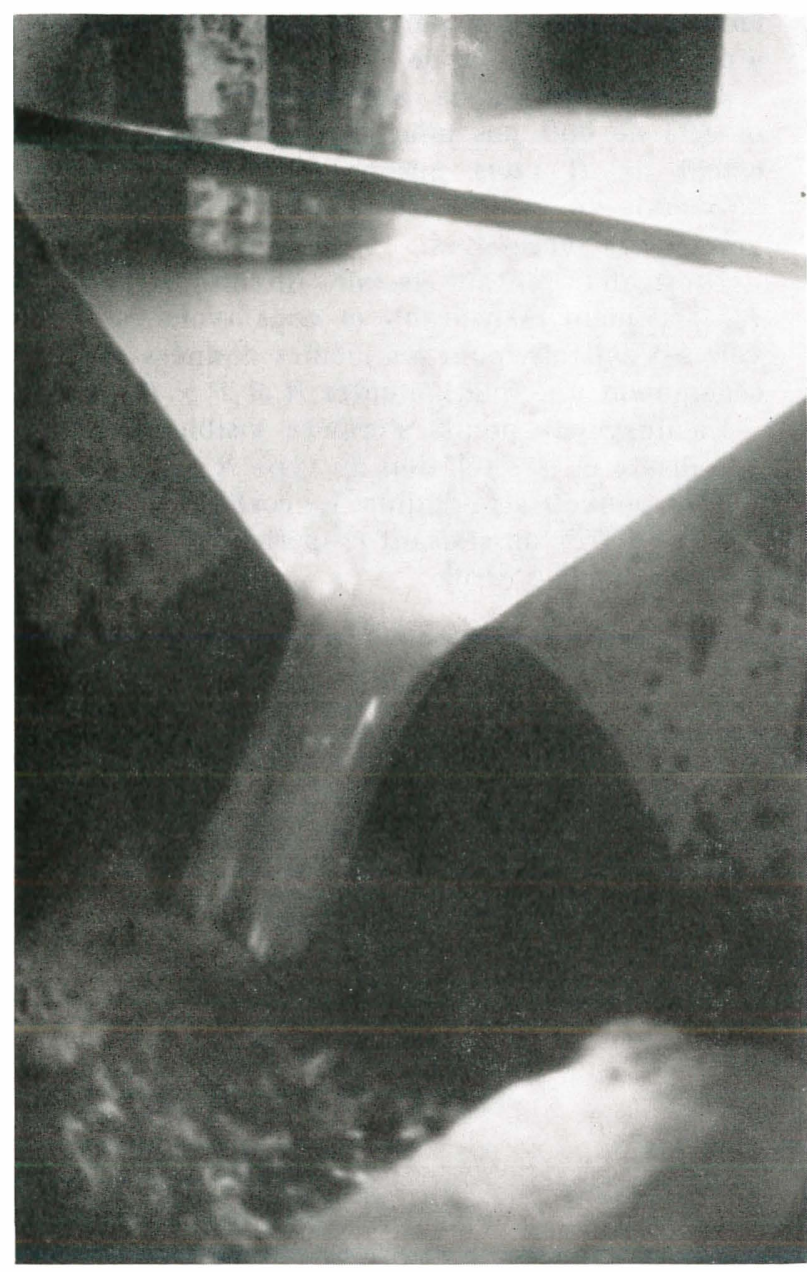

Photo Galabert.

Un autre type de cuve utilisé à Allokoto. suffit pour ce faire de disposer des pluviogrammes entreposés dans les capitales de ces Etats et on obtiendrait ainsi rapidement une carte de facteur $R$.

En Haute-Volta, J. GALABERT a vérifié la validité de notre équation (1) à partir de nouvelles données : 119 couples des stations de Dori (14º lat. $\mathrm{N}, 0^{\mathrm{e}}$ long., P. moyenne, $587 \mathrm{~mm}$ ) et Bobo-Dioulasso (110 10' lat. N, 40 20 long. P. moyenne : $1.160 \mathrm{~mm}$ ) et il a obtenu l'équation

$$
R=0,015884 P I_{30}-1,24
$$

équation très voisine de l'équation (1). Nous proposons pour la zone sahélo-soudanienne l'adoption de la formule moyenne

$$
\text { - } R=0,0158 \quad P I_{30}-1,2 \text { (3) }
$$

très satisfaisante pour des points aussi éloignés en distance $(1.100 \mathrm{~km})$ qu'en pluviométrie $(440 \mathrm{~mm}$ et $1.160 \mathrm{~mm})$.

Nous avons pu calculer à partir de ces équations les valeurs de $R$ annuelles pour un certain nombre de stations. Nous donnons à titre d'exemple celles calculées par J. GALABERT pour la Haute-Volta à l'aide de l'équation 2 : tableau 8. Malheureusement il s'est révélé que peu de stations disposaient d'un pluviographe à rotation journalière, et celles qui en possédaient l'utilisaient depuis peu de temps. La majorité des pluviographes équipant les stations sont à rotation hebdomadaire ce qui interdit la lecture du facteur $I_{30}$. Il ne nous est donc pas possible pour l'instant de dresser une carte du facteur $R$ pour l'ensemble des deux Etats, le nombre de stations étant trop faible et les moyennes actuelles ne portant que sur des périodes limitées (neuf ans pour Maradi, Niamey et généralement beaucoup moins : 4 ou 5 ans).

TABLEAU 8

\begin{tabular}{|c|c|c|c|c|c|c|c|c|}
\hline Station ${ }^{\text {Année }}$ & 1966 & 1967 & 1968 & 1969 & 1970 & 1971 & 1972 & Moyenne \\
\hline 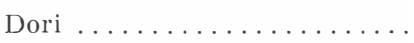 & 440 & 190 & 176 & 491 & 165 & 101 & 262 & 261 \\
\hline Ouahigouya.............. & & 390 & 315 & 353 & 225 & 321 & 203 & 301 \\
\hline Fada N'Gourma ............. & 442 & 526 & 439 & 458 & 302 & 322 & 509 & 428 \\
\hline Ouagadougou & & 389 & 252 & 725 & 228 & 414 & 786 & 466 \\
\hline Saria $\ldots \ldots \ldots \ldots \ldots \ldots$ & & & 357 & 501 & 389 & 320 & 217 & 357 \\
\hline$\ldots \ldots \ldots \ldots \ldots \ldots$ & & & 406 & 467 & 384 & 256 & 378 & 378 \\
\hline Bobo-Dioulasso & 493 & 437 & 664 & 538 & 880 & 421 & 442 & 554 \\
\hline Farako-Ba $\ldots \ldots \ldots \ldots \ldots$ & & 365 & 393 & 489 & 736 & 372 & 554 & 485 \\
\hline Niangoloko $\ldots \ldots \ldots \ldots \ldots$ & & & 707 & 846 & 924 & 314 & 491 & 656 \\
\hline Gaoua & 419 & 274 & 910 & 644 & 713 & 496 & 310 & 538 \\
\hline
\end{tabular}

Valeur de $R$ en Haute- Volta 


\section{LE RUISSELLEMENT}

Le ruissellement est néfaste à deux points de vue : il constitue une perte en eau inadmissible dans des sols à bilan hydrique déficitaire sous des pluviométries marginales, et il est générateur et transporteur d'érosion. C'est pour cette raison que nous étudions de façon détaillée le ruissellement. Le dispositif expérimental choisi nous permet des observations instantanées sur le ruissellement au cours d'une crue, et d'apprécier donc son intensité, que l'on peut comparer aisément à l'intensité pluviométrique.

La figure 9 relative à la pluie du 16 juillet 67 donne les intensités pluviométriques et de ruissellement sur les parcelles P1, P2 et P3. On y constate :

- la rapidité du déclenchement du ruissellement suite à la pointe de l'averse : le déclenchement est presqu'instantané ;

- le décalage entre pointe de crue et pointe de l'averse est de l'ordre de 5 à $10 \mathrm{mn}$;

- la réponse des parcelles traitées est toujours plus lente que celle de la parcelle témoin, les pointes sont fortement écrêtées mais le ressuyage de ces parcelles est plus lent.

Ces constatations effectuées sur un exemple ont un caractère très général.

Les ruissellements ont été exprimés par le coefficient de ruissellement qui est le rapport du volume ruisselé au volume de pluie tombée sur la parcelle. Mais afin de comparer les intensités de ruissellement des parcelles entre elles, on a exprimé les pointes de ruissellement en litres par seconde et par hectare.

A titre d'exemple le tableau 9 donne les valeurs recueillies en 1967 pour les pluies ayant ruisselé.

L'examen de ce tableau permet de dégager un certain nombre d'observations :

- Il n’y a ruissellement qu'à partir d'une certaine hauteur d'eau tombée. En ce qui concerne la parcelle témoin $\mathrm{P} 2$, ce ruissellement semble se produire à partir de pluies de l'ordre de $12 \mathrm{~mm}$ (pluie du 4 août) ce qui justifie que le facteur $R$ ne soit calculé qu'à partir de $1 / 2$ pouce $(12,7 \mathrm{~mm})$.

- Cette condition n'est cependant pas suffi-

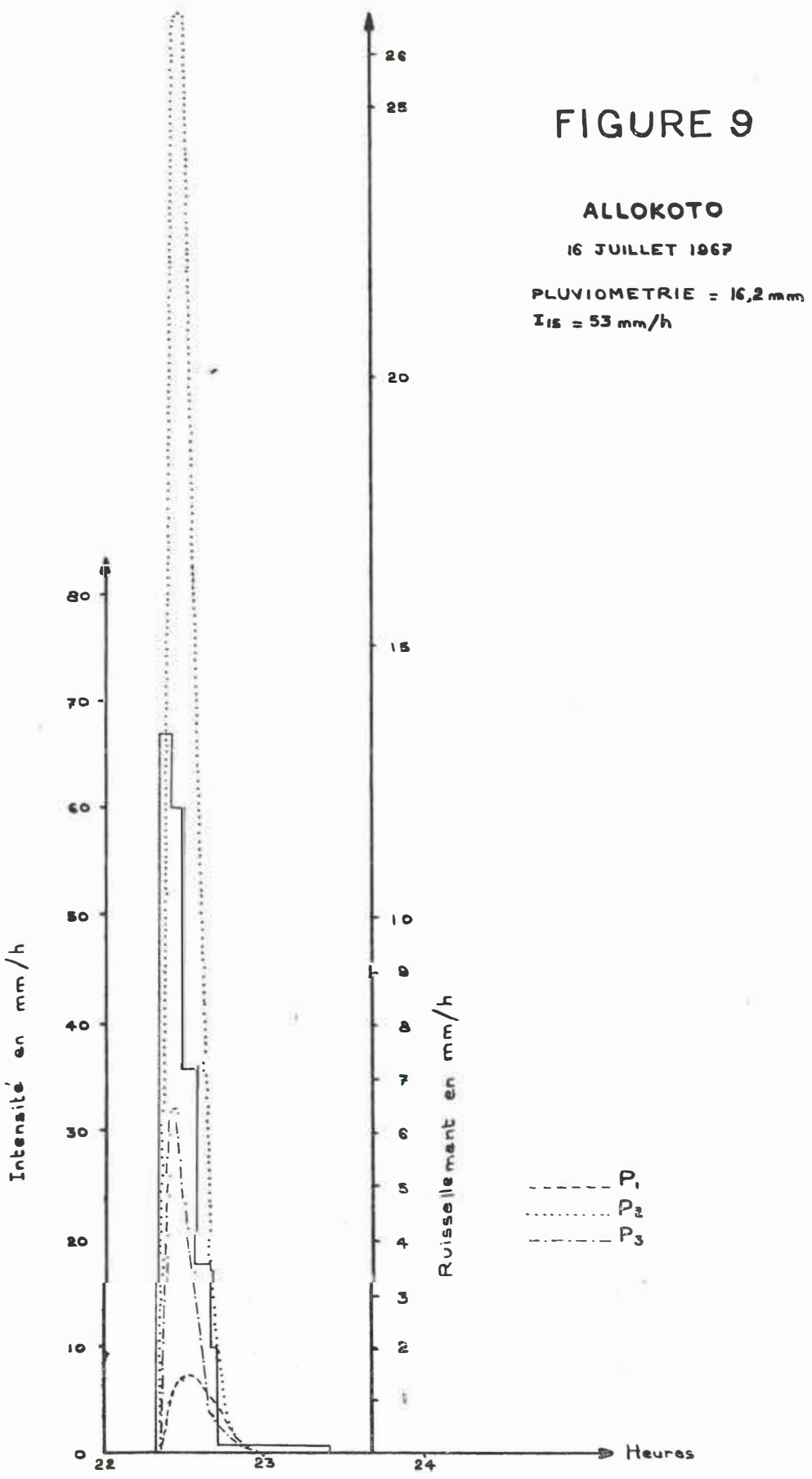

sante et nous avons volontairement fait figurer dans le tableau trois pluies qui quoique supérieures à $12 \mathrm{~mm}$ n'ont donné lieu à aucun ruissellement $(10 / 8,24 / 8,24 / 8)$. Ces pluies sont en effet caractérisées par un facteur $R$ relativement bas : elles sont peu érosives et n'ont en l'occurrence occasionné aucune érosion. 
TABLEAU 9

Allokoto, 1967, Ruissellements journaliers

\begin{tabular}{|c|c|c|c|c|c|c|c|c|c|c|c|c|}
\hline \multirow{2}{*}{ Date } & \multirow{2}{*}{$\begin{array}{l}\text { Pluviométrie } \\
\text { en mm }\end{array}$} & \multirow{2}{*}{$\begin{array}{l}\text { Intensité } \\
\max \text {. } \\
\text { en } \mathrm{mm} / \mathrm{h}\end{array}$} & \multirow{2}{*}{$\begin{array}{l}\text { Intensité } \\
\text { en } 15^{\prime} \\
\text { en } \mathrm{mm} / \mathrm{h}\end{array}$} & \multirow{2}{*}{$R$} & \multicolumn{4}{|c|}{ Ruissellement en \% } & \multicolumn{4}{|c|}{$\begin{array}{l}\text { Pointe de ruissellement } \\
\text { en } 1 / \mathrm{s} / \mathrm{ha}\end{array}$} \\
\hline & & & & & P1 & P2 & P3 & $\mathrm{P} 4$ & P1 & P2 & P3 & $\mathrm{P} 4$ \\
\hline $\begin{array}{c}\text { Juin : } \\
23 \ldots \ldots \ldots \\
27 \ldots \ldots \\
27 \ldots \ldots \\
29 \ldots \ldots\end{array}$ & $\begin{array}{r}24,8 \\
23,6 \\
10,4 \\
6,7\end{array}$ & $\begin{array}{l}84 \\
66 \\
36 \\
20\end{array}$ & $\begin{array}{l}54 \\
60 \\
18 \\
16\end{array}$ & $\begin{array}{l}17,34 \\
10,94 \\
- \\
-\end{array}$ & E & $\begin{array}{r}8,8 \\
16,8 \\
24,0 \\
8,5\end{array}$ & E & - & E & $\begin{array}{r}7,3 \\
26,3 \\
8,3 \\
4,0\end{array}$ & E & E \\
\hline $\begin{array}{l}\text { Juillet : } \\
15 \quad \ldots \ldots \\
16 \\
23 \\
23 \\
23 \\
24 \\
24 \ldots\end{array}$ & $\begin{array}{l}23,3 \\
16,2 \\
44,1 \\
15,7 \\
31,0\end{array}$ & $\begin{array}{r}108 \\
104 \\
96 \\
84 \\
132\end{array}$ & $\begin{array}{l}63 \\
53 \\
88 \\
56 \\
88\end{array}$ & $\begin{array}{r}16,19 \\
7,58 \\
56,85 \\
7,61 \\
24,92\end{array}$ & $\begin{array}{l}\begin{array}{l}1,95 \\
3,6 \\
20 \\
11,1 \\
24,7\end{array} .\end{array}$ & $\begin{array}{l}29,6 \\
34,4 \\
59,5 \\
70 \\
51,3\end{array}$ & $\begin{array}{r}0,7 \\
8,6 \\
14,6 \\
23,5 \\
37,2\end{array}$ & $\begin{array}{c}- \\
- \\
-\end{array}$ & $\begin{array}{l}0,46 \\
2 \\
45 \\
14,8 \\
39,1\end{array}$ & $\begin{array}{r}45,9 \\
25,6 \\
151 \\
126,7 \\
180,0\end{array}$ & $\begin{array}{l}\overline{8,6} \\
94 \\
54 \\
91,0\end{array}$ & $\begin{array}{l}\text { E } \\
\text { - } \\
\text { - }\end{array}$ \\
\hline 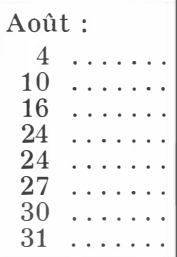 & $\begin{array}{r}12,4 \\
12,6 \\
15,8 \\
19,3 \\
15,7 \\
20,8 \\
35,0 \\
8,8\end{array}$ & $\begin{array}{r}75 \\
66 \\
90 \\
75 \\
42 \\
84 \\
150 \\
30\end{array}$ & $\begin{array}{l}38 \\
40 \\
62 \\
46 \\
30 \\
48 \\
82 \\
12\end{array}$ & $\begin{array}{r}\overline{4,28} \\
7,74 \\
6,73 \\
3,20 \\
8,71 \\
21,50 \\
-\end{array}$ & $\begin{array}{l}- \\
- \\
\bar{E} \\
\overline{4} \\
-\end{array}$ & $\begin{array}{c}11,4 \\
\overline{17,8} \\
- \\
- \\
1,4 \\
9,5 \\
3,2\end{array}$ & $\begin{array}{l}- \\
\bar{z} \\
\bar{z} \\
\overline{1,4}\end{array}$ & $\begin{array}{l}- \\
- \\
- \\
-\end{array}$ & $\begin{array}{l}- \\
- \\
\bar{E} \\
\bar{E}\end{array}$ & $\begin{array}{r}26,1 \\
\overline{72,5} \\
- \\
\overline{2}, 4 \\
74,8 \\
2,4\end{array}$ & $\begin{array}{l}- \\
\bar{z} \\
\bar{z} \\
\bar{z}, 2\end{array}$ & $\begin{array}{l}\text { - } \\
\text { - } \\
\text { - } \\
\text { - }\end{array}$ \\
\hline $\begin{array}{r}\text { Septembre : } \\
7 \ldots \ldots \ldots \\
13 \quad \ldots \ldots \ldots \\
24 \quad \ldots \ldots \ldots\end{array}$ & $\begin{array}{l}18,9 \\
63,5 \\
23,3\end{array}$ & $\begin{array}{r}66 \\
102 \\
40\end{array}$ & $\begin{array}{l}52 \\
98 \\
28\end{array}$ & $\begin{array}{r}7,63 \\
98,67 \\
5,94\end{array}$ & $\underline{15,0}$ & $\begin{array}{r}17,3 \\
49,1 \\
7,0\end{array}$ & $\overline{6,8}$ & - & $\underline{46,5}$ & $\begin{array}{r}38,6 \\
119,0 \\
11,3\end{array}$ & $\overline{39,0}$ & 二 \\
\hline
\end{tabular}

- Une pluie tombant sur un sol gorgé d'eau ruisselle beaucoup plus. Ceci est particulièrement évident lorsque deux pluies se succèdent au cours de la même journée (27 juin, 23 juillet) et le ruissellement peut alors se produire pour des pluies d'assez faible hauteur $(10,4 \mathrm{~mm}$ le 27 juin). Inversement, une pluie tombant sur un sol à peu près sec ruisselle peu. C'est ce qu'on constate avec la pluie du 23 juin qui, quoiqu'assez érosive, a assez peu ruisselé.

- Les pluies de juin-juillet (début de la saison des pluies) ruissellent plus fortement que celles de fin de saison des pluies (août-septembre). Ceci est dû pour une part au fait que les premières pluies sont généralement plus érosives que les suivantes ( $R$ et $I_{\max }$ plus importants) et également au fait que la couverture végétale, de plus en plus fournie, freine le ruissellement à partir de fin juillet.

- Les parcelles P1, P3 ont beaucoup moins ruisselé que la $\mathrm{P} 2$ et la parcelle $\mathrm{P} 4$ n'a absolument jamais ruisselé. Ceci est dû aux dispositifs antiérosifs mais nous le constaterons mieux sur les tableaux 10 et 11 suivants.

Le tableau 10 donne la répartition des coefficients et pointes de ruissellement par classes pour chacune des parcelles au cours des six années d'observations. Ce tableau permet de constater que les coefficients de ruissellement peuvent dépasser $50 \%$ pour la parcelle témoin (maximum absolu enregistré $70 \%$ le 23 juillet 1967 pour une pluie de $15,7 \mathrm{~mm}$ succédant à une première pluie le même jour de $44,1 \mathrm{~mm}$ ) alors qu'ils ne dépassent jamais la classe $20-30 \%$ pour les parcelles 1 et 3 (respectivement murets et lignes de végétation) et qu'ils sont limités à la première classe $(0-10 \%)$ en ce qui concerne la parcelle 4 (bourrelets armés).

Les trois dispositifs en place ont donc fortement freiné le ruissellement. La quantité d'eau emmagasinée par les sols a donc été plus forte et les rendements des cultures doivent s'en ressentir. Il en ira de même bien sûr pour les pertes en terre.

Le tableau 11 enfin donne les pourcentages moyens de ruissellement enregistrés. Les différences entre parcelles s'y retrouvent. Notons que le dispositif antiérosif le plus rudimentaire que nous ayions mis en place ( $\mathrm{P} 3$, lignes de végétation isohypses) permet de faire passer le ruissellement de $17,6 \%$ à $5,2 \%$.

Notons aussi que les murets (P1), qui ont une efficacité moindre que les bourrelets armés (P4) maintiennent et peut-être même renforcent cette efficacité dans le temps alors que dans le cas des bourrelets armés, il semble y avoir une tendance à l'augmentation du ruissellement avec le temps : Ceci est dû pour le premier cas à l'atterrissement progressif se concrétisant par la formation de terrasses, formation existant également dans le 
TABLEAU 10

Allokoto, Répartition des coefficients et pointes de ruissellement par classes

\begin{tabular}{|c|c|c|c|c|c|c|c|c|c|c|c|c|c|c|c|c|c|c|c|c|c|c|c|c|}
\hline \multirow{9}{*}{ 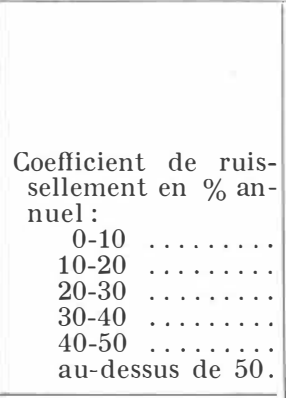 } & \multicolumn{6}{|c|}{ Parcelle $n^{\circ} 1$} & \multicolumn{6}{|c|}{ Parcelle $n^{\circ} 2$} & \multicolumn{6}{|c|}{ Parcelle $n^{\circ} 3$} & \multicolumn{6}{|c|}{ Parcelle $n^{\circ} 4$} \\
\hline & 66 & 67 & 68 & 69 & 70 & 71 & 66 & 67 & 68 & 69 & 70 & 71 & 66 & 67 & 68 & 69 & 70 & 71 & 66 & 67 & 68 & 69 & 70 & 71 \\
\hline & & & & & & & & & & & & & & & & & & & $\stackrel{\stackrel{0}{Z}}{?}$ & & & & & \\
\hline & 6 & 3 & 1 & 3 & 9 & 13 & 8 & 6 & 6 & 5 & 7 & 6 & 3 & 4 & $\overline{1}$ & 2 & 3 & 11 & 氙 & - & 1 & 5 & 7 & 6 \\
\hline & 1 & 2 & - & 1 & 2 & 0 & 2 & 4 & - & 6 & 2 & 3 & 4 & 1 & 1 & 2 & 5 & - & & - & - & - & - & - \\
\hline & 3 & 2 & - & 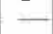 & 2 & 0 & 1 & 2 & 1 & 3 & 2 & 3 & 3 & 1 & - & $\overline{2}$ & 2 & - & $\tilde{ช}$ & - & - & - & - & 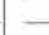 \\
\hline & - & - & - & - & - & 0 & 3 & 1 & - & 3 & 4 & 2 & - & - & - & - & - & - & $\Xi$ & - & - & - & - & - \\
\hline & - & - & - & - & - & 0 & 4 & 1 & - & 3 & 1 & - & - & - & - & - & - & - & $\dot{Z}$ & - & - & - & - & - \\
\hline & - & - & 一 & - & - & - & 1 & 3 & 1 & - & 1 & - & - & - & - & - & - & - & & - & - & - & 一 & - \\
\hline Pointe de ruisselle- & & & & & & & & & & & & & & & & & & & & & & & & \\
\hline $\begin{array}{c}\text { ment } 1 / \text { s/ha }: \\
0-20 \ldots \ldots \ldots\end{array}$ & 5 & 4 & 1 & 2 & 7 & 1 & 4 & 6 & 6 & 4 & 5 & 5 & 3 & 3 & - & 1 & 2 & 2 & & - & - & 1 & 7 & 1 \\
\hline $20-40 \ldots \ldots \ldots$ & 1 & 1 & - & 1 & 1 & - & - & 4 & - & 5 & 2 & 1 & 5 & 5 & 1 & 1 & 2 & 1 & $\stackrel{\leftrightarrow}{.}$ & - & 1 & 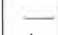 & - & 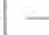 \\
\hline $40-60 \ldots \ldots \ldots$ & 2 & 2 & - & 一 & - & - & 2 & 1 & - & 2 & 3 & 4 & 1 & 1 & 1 & 更 & 2 & - & 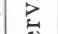 & - & - & 1 & - & - \\
\hline $60-80 \ldots \ldots \ldots$ & - & - & 一 & 一 & - & - & 2 & 2 & - & & $\overline{0}$ & - & 1 & - & - & 2 & 2 & 一 & ¿ & - & - & - & - & - \\
\hline $\begin{array}{r}80-100 \\
100-120\end{array}$ & - & - & - & - & - & - & 2 & 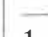 & $\overline{1}$ & 2 & 2 & 1 & - & 2 & - & - & - & E & है & - & - & - & - & 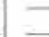 \\
\hline $120-140 \quad \ldots \ldots \ldots$ & - & - & - & - & - & - & 1 & $\begin{array}{l}1 \\
1\end{array}$ & 1 & 1 & $\begin{array}{l}1 \\
1\end{array}$ & 1 & - & 二 & - & - & - & - & ร & 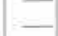 & - & - & - & - \\
\hline $140-160 \ldots \ldots$ & - & - & - & - & - & - & 1 & 1 & 1 & 1 & - & - & - & - & - & - & - & $一$ & $\stackrel{0}{\mathrm{z}}$ & - & - & - & 一 & - \\
\hline $160-180 \quad \ldots \ldots \ldots$ & 一 & - & - & - & - & - & - & 1 & - & - & - & - & - & - & - & - & - & - & & - & - & - & - & - \\
\hline
\end{tabular}

TABLEAU 11

Allokoto, Pourcentages moyens de ruissellement enregistrés

\begin{tabular}{|c|c|c|c|c|c|c|}
\hline Année & Pluviométrie & $R$ & $\mathrm{P} 1$ & $\mathrm{P} 2$ & P3 & $\mathrm{P} 4$ \\
\hline $1966 \ldots \ldots \ldots \ldots \ldots$ & 487,0 & 180,4 & 5,1 & 18 & 6,1 & $(*)$ \\
\hline $1967 \ldots \ldots \ldots \ldots \ldots$ & 515,3 & 305,8 & 5,9 & 22,5 & 5,0 & 0 \\
\hline $1968 \ldots \ldots \ldots \ldots \ldots$ & 354,3 & 150,8 & 0,8 & 8,5 & 1,2 & 0,6 \\
\hline $1969 \ldots \ldots \ldots \ldots$ & 506,8 & 178,0 & 2,3 & 21,4 & 6,8 & 1,2 \\
\hline $1970 \ldots \ldots \ldots \ldots$ & 469,5 & 250,9 & 5,6 & 19,2 & 8,5 & 2,1 \\
\hline $1971 \ldots \ldots \ldots \ldots$ & 289,6 & 152,6 & 1,3 & 10,5 & 1,3 & 0,3 \\
\hline Moyenne $\ldots \ldots \ldots \ldots$ & 437,0 & 198,5 & 3,8 & 17,6 & 5,2 & 0,9 \\
\hline
\end{tabular}

(*) Non en service.

second cas mais perturbée par des dégradations d'un système plus fragile. Des dégradations du même ordre se ressentent dans la parcelle 3 .

Si nous examinons le tableau par années, nous constatons que nous avons deux années très peu érosives avec $R$ voisin de 150 (1968 et 1971), deux années assez peu érosives $R$ voisin de 180 (1966 et 1969) et deux années érosives (1970, $R=250$;
1967, $R>300$ ). Ces différences se retrouvent approximativement dans les valeurs de ruissellement avec une exception cependant : Les valeurs du ruissellement en 1966 pour P1 et P3 sont relativement trop fortes, cela doit être imputé au fait que nous sommes en première année d'installation et aucun atterrissement ni colmatage n'a encore eu lieu.

\section{VI. - L'ÉROSION}

Les pertes en terre sont constituées

- par des dépôts de cuve,

- par les matières en suspension.
Pour les premières, on recueille après les pluies quatre échantillons de boue dans chaque cuve après floculation et siphonage de l'eau en notant la hauteur d e boue. Il est alors aisé après dessiccation 


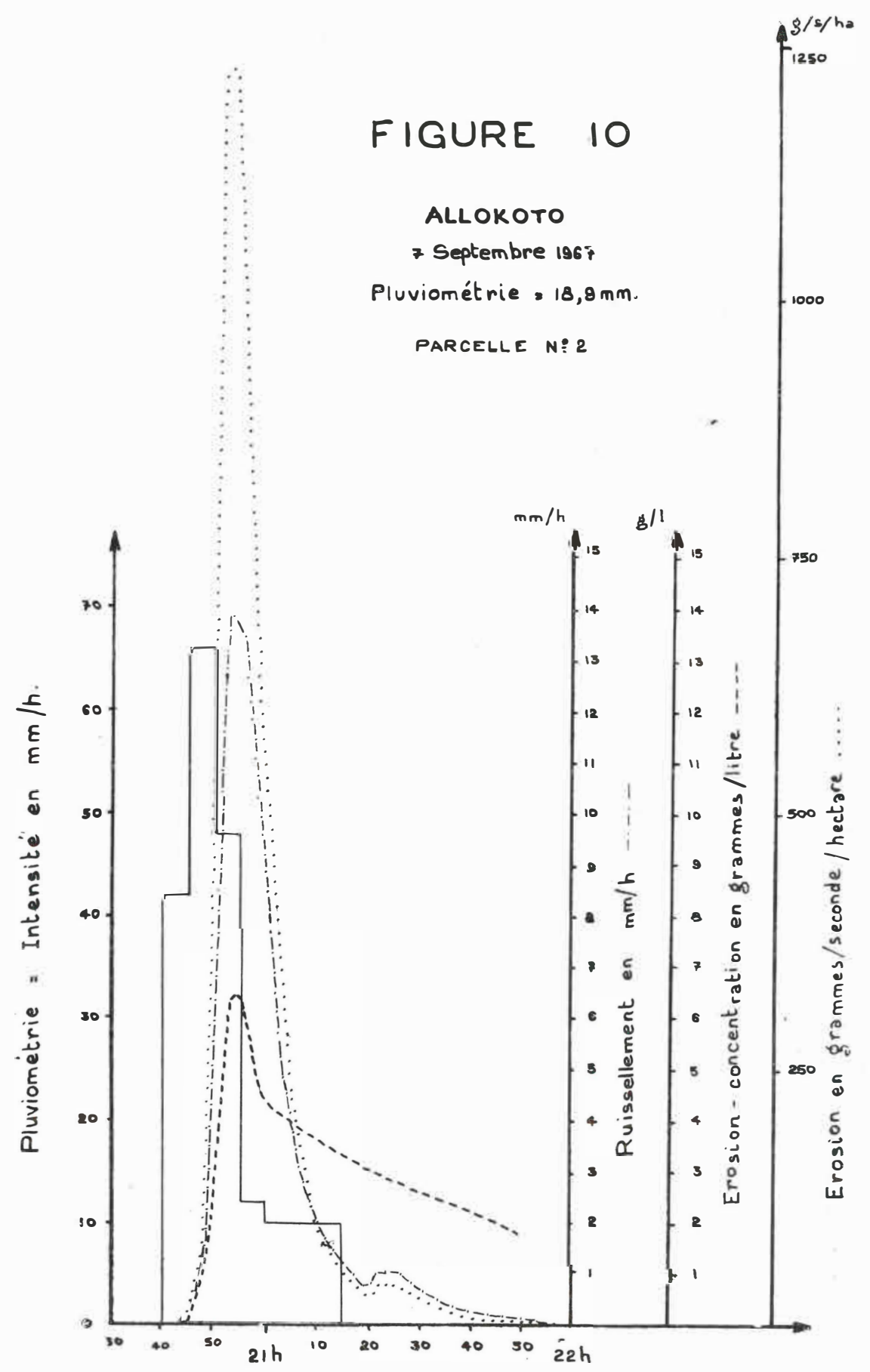

limnigramme et on en déduit la courbe des débits solides en grammes par seconde. Par planimétrage on obtient la quantité totale de matière sèche exportée au cours d'une pluie. Cette manière de procéder entraîne cependant une imprécision sur le chiffre final ceci pour deux raisons :

- Il n'est pas toujours facile de placer le point exact de prise d'échantillon sur le limnigramme surtout lorsque celui-ci est en brusque montée ou descente.

- On a rarement une prise d'échantillon au sommet du limnigramme qui est le point de ruissellement maximum et on est amené à effectuer une interpolation sur la concentration en ce point, interpolation qui peut avoir des conséquences importantes vu le débit.

A titre d'exemple, la figure 10 donne la représentation de l'érosion sur la parcelle 2 au cours d'une pluie (7 septembre 1967). Cette pluie est représentative, on $\mathrm{y}$ constate que :

- La pointe de ruissellement suit de $7 \mathrm{mn}$ la pointe d'intensité maximum, nous avions déjà signalé cet écart.

- A la pointe de ruissellement maximum correspond une concentration maximum des eaux en terre et corrélativement la pointe des débits solides correspond également.

- Les trois courbes ruissellement, concentration et

en laboratoire de connaître la quantité totale de matière sèche ainsi recueillie au cours des pluies. Les valeurs trouvées sont satisfaisantes.

Pour les secondes, on effectue toutes les cinq minutes un prélèvement d'un litre au déversoir des cuves en commençant dès le premier écoulement. Ces échantillons, soigneusement étiquetés, sont traités au laboratoire et on connaît donc toutes les cinq minutes la concentration en matière sèche des eaux écoulées. Ces valeurs sont reportées sur le débits solides varient sensiblement dans le même sens.

Ces constatations ont un caractère très général.

Ces analyses des pertes en terre ont eu lieu tous les ans sauf en 1966 ou seuls les dépôts de cuves ont été relevés.

Par ailleurs, pour les autres années, il nous est arrivé de ne pouvoir disposer de tous les renseignements nécessaires pour certaines pluies (manœuvre 
TABLEAU 12

Allokoto, 1968, Etude des pluies érosives par parcelles

\begin{tabular}{|c|c|c|c|c|c|c|c|c|c|c|c|c|c|c|c|}
\hline \multirow{2}{*}{ Date } & \multirow{2}{*}{$\begin{array}{l}\text { Pluvio- } \\
\text { métrie } \\
\text { en mm }\end{array}$} & \multirow{2}{*}{$\mathrm{mm}_{15} / \mathrm{h}$} & \multirow{2}{*}{$\begin{array}{l}\text { Index } \\
\quad R\end{array}$} & \multicolumn{4}{|c|}{ Dépôts lourds en kg/ha } & \multicolumn{4}{|c|}{ Suspensions kg/ha } & \multicolumn{4}{|c|}{ Total par pluie } \\
\hline & & & & $\mathrm{P} 1$ & $\mathrm{P} 2$ & P3 & $\mathrm{P} 4$ & $\mathrm{P} 1$ & P2 & P3 & $\mathrm{P} 4$ & $\mathrm{P} 1$ & $\mathrm{P} 2$ & P3 & P 4 \\
\hline $\begin{array}{ll}11-6 & \ldots \\
16-6 & \ldots \\
20-6 & \ldots \\
29-6 & \ldots \\
\end{array}$ & $\begin{array}{r}7,0 \\
13,3 \\
12,6 \\
11,0\end{array}$ & $\begin{array}{l}\overline{26} \\
46 \\
18\end{array}$ & $\begin{array}{l}- \\
3,37 \\
4,88 \\
1,50\end{array}$ & & $\begin{array}{r}66 \\
105 \\
148 \\
123\end{array}$ & & & & & & & & $\begin{array}{r}66 \\
105 \\
148 \\
123\end{array}$ & & \\
\hline \begin{tabular}{ll|}
$18-7$ & $\ldots$ \\
$26-7$ & $\cdots$
\end{tabular} & $\begin{array}{l}20,6 \\
15,8\end{array}$ & $\begin{array}{l}56 \\
36\end{array}$ & $\begin{array}{l}9,21 \\
4,18\end{array}$ & & $\begin{array}{r}162 \\
97\end{array}$ & & & & $\begin{array}{l}8 \\
3\end{array}$ & & & & $\begin{array}{l}170 \\
100\end{array}$ & & \\
\hline \begin{tabular}{ll|}
$18-8$ & $\ldots$ \\
$20-8$ & $\ldots$
\end{tabular} & $\begin{array}{l}35,2 \\
32,0\end{array}$ & $\begin{array}{l}96 \\
94\end{array}$ & $\begin{array}{l}31,8 \\
31,45\end{array}$ & $\begin{array}{r}1.176 \\
66\end{array}$ & 737 & 85 & 107 & 75 & $\begin{array}{l}1.500 \\
1.030\end{array}$ & 328 & 78 & 141 & $\begin{array}{l}2.676 \\
1.737\end{array}$ & 413 & 185 \\
\hline \multicolumn{3}{|c|}{$\begin{array}{l}\text { Index } \mathrm{R} \text { pour l'année et total des } \\
\text { pertes en terre en t/ha/année } \ldots . . .\end{array}$} & 150,57 & 0,066 & 2,614 & 0,085 & 0,107 & 0,075 & 2,541 & 0,328 & 0,078 & 0,141 & 5,125 & 0,413 & 0,185 \\
\hline
\end{tabular}

absent lors de la pluie, bouteille cassée lors du transport) ce qui fait que les chiffres globaux perdent leur signification. Nous préférons pour cette raison fournir le tableau 12 qui donne pour 1968 les valeurs recueillies, cette année ayant été choisie du fait que nous possédons pour elle toutes les données. Si on examine ce tableau, nous constatons que malgré le fait que 1968 soit une année peu érosive $(R=$ $150,6)$ on n'en obtient pas moins une perte en terre de plus de 5 t/ha pour la parcelle travaillée traditionnellement, érosion qui est en fait causée essentiellement par deux pluies seulement : celles du 18 et du 20 août totalisant à elles deux une exportation de 4,4 t. L'érosion sur les parcelles traitées est par contre insignifiante et toujours inférieure à $500 \mathrm{~kg} / \mathrm{ha}$.

Cette constatation est assez générale : En 1967 trois pluies, celles des 23/7 $(R=56,8), 24 / 7(R=$ $24,9)$ et $13 / 9(R=98,7)$ sont responsables d'une perte de plus de 5,7 t/ha uniquement en suspension (parcelle P2).

En 1969 la pluie du 5 juillet $(R=27,9)$ fait perdre $3 \mathrm{t} /$ ha à la même parcelle et celle du 5 août $(R=28,6) 2,1 \mathrm{t} /$ ha. Il en va de même au cours des années 70 (1,5 t/ha le 31 juillet $)$ et en $71(1,9 \mathrm{t}$ le 13 juillet).

Nous avons donné au tableau 13 les ordres de grandeur des pertes annuelles enregistrées. Ce tableau nous confirme que le dispositif antiérosif de P4 (bourrelets armés) est le plus efficace avec cependant une légère tendance à la dégradation. Les murets sont eux-mêmes très efficaces (P11) alors que les lignes de végétation isohypses ont une efficacité moindre. Quant aux chiffres, ils suivent d'une manière assez satisfaisante les valeurs de $R$, l'érosion la plus intense étant obtenue en 1967 $(R=305,8$ pour une perte de plus de $18 \mathrm{t}$ en $\mathrm{P} 2)$ et les plus faibles en 68 et $71(R \sim 150)$ avec respectivement 5,0 t et 3,5 t en P2 : Cette dernière différence se justifie en fait par une pluviométrie de nature différente (cf. tabl. 3 - 1968: 4 pluies entre
30 et $50 \mathrm{~mm}, 1971$ : pas de pluie supérieure à 30 ) et des valeurs de $R$ plus modiques dans le second cas ( $R$ dépasse deux fois 30 en 68 alors qu'il n'atteint jamais 25 en 71 ).

TABLEAU 13

Allokoto, Pertes en terre

\begin{tabular}{|c|c|c|c|c|c|c|}
\hline \multirow[b]{3}{*}{1967} & \multirow{3}{*}{$\begin{array}{l}P / \mathrm{mm} \\
515,3\end{array}$} & \multirow{3}{*}{$\begin{array}{c}R \\
305,8\end{array}$} & \multicolumn{4}{|c|}{ Pertes en terre en $t$ /ha } \\
\hline & & & $\mathrm{P} 1$ & $\mathrm{P} 2$ & P3 & P4 \\
\hline & & & 1,5 & 18,5 & 3,0 & - \\
\hline 1968 & 354,3 & 150,8 & 0,1 & 5,0 & 0,5 & 0,2 \\
\hline 1969 & 506,8 & 178,0 & 0,5 & 11,0 & 1,0 & 0,2 \\
\hline 1970 & 469,5 & 250,9 & 0,5 & $X$ & 0,8 & 0,5 \\
\hline 1971 & 289,6 & 152,6 & 0,1 & 3,5 & 0,2 & 0,1 \\
\hline
\end{tabular}

$X$ : Chiffre trop imprécis pour être cité.

La comparaison entre les différentes parcelles doit être complétée par la comparaison entre les concentrations moyennes des eaux de ruissellement (turbidité). Les valeurs annuelles moyennes varient entre :

4 et $5 \mathrm{~g} / \mathrm{l}$ pour la parcelle 1 .

13 à $18 \mathrm{~g} / \mathrm{l}$ pour la parcelle 2 .

6 à $11 \mathrm{~g} / \mathrm{l}$ pour la parcelle 3 .

8 à $10 \mathrm{~g} / \mathrm{l}$ pour la parcelle 4 .

Ces variations de concentration entre parcelles expliquent le non-parallélisme des valeurs du ruissellement et de l'érosion entre parcelles. Singulièrement, la parcelle témoin qui enregistre les ruissellements les plus importants avec des concentrations doubles ou triples des autres parcelles enregistre des pertes en terre considérables. Il faut également souligner le rôle des murets de pierre sèche dans le filtrage des éléments. 


\section{VII. - ROLE DE LA CULTURE, DU TRAVAIL ET DE LA NATURE DU SOL, DE L'ËTAT DE LA VÉGÉTATION}

1) Cultures.

Les cultures qui se sont succédées à Allokoto sont les suivantes :

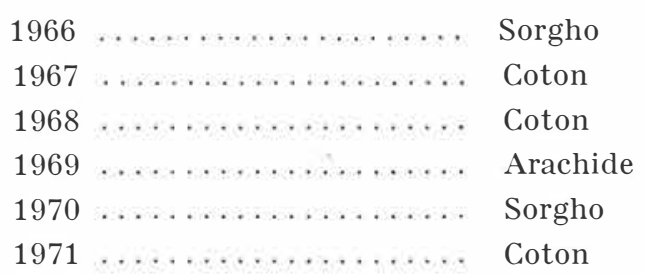

La pluviométrie des années 1968 et 1971 (354 et $290 \mathrm{~mm}$ ) était nettement insuffisante pour obtenir une récolte correcte et les rendements ont été effectivement dérisoires.

Les autres années, avec des pluviométries importantes et supérieures à $450 \mathrm{~mm}$, ont permis des cultures correctes encore que les attaques de Striga sur le Sorgho aient un peu faussé les résultats des années 1966 et 70. Nous donnons dans le tableau 14 les résultats obtenus sur coton et arachide en 67 et 69 , années les plus pluvieuses. Ce tableau montre que les sols testés peuvent porter ces cultures d'une manière intéressante. Il est d'ailleurs encourageant de constater que les années suivantes, le chef de village, suivi par des paysans a entrepris ces cultures sur vertisols.

\section{TABLEAU 14}

Allokoto, Rendement des cultures

1967, Coton : $P=515,3 \mathrm{~mm}$

\begin{tabular}{|c|c|c|}
\hline Parcelle & $\begin{array}{c}\text { Poids total } \\
\text { kg/ha }\end{array}$ & $\begin{array}{c}\text { Gain par rapport } \\
\text { témoin \% }\end{array}$ \\
\cline { 2 - 3 } & 800 & 95,1 \\
2 & 410 & 0 \\
3 & 810 & 97,5 \\
4 & 1.200 & 192,6 \\
\hline
\end{tabular}

Allokoto, février 1951, un autre aménagement anti-érosif par construction progressive de terrasses pour réaliser des murets.

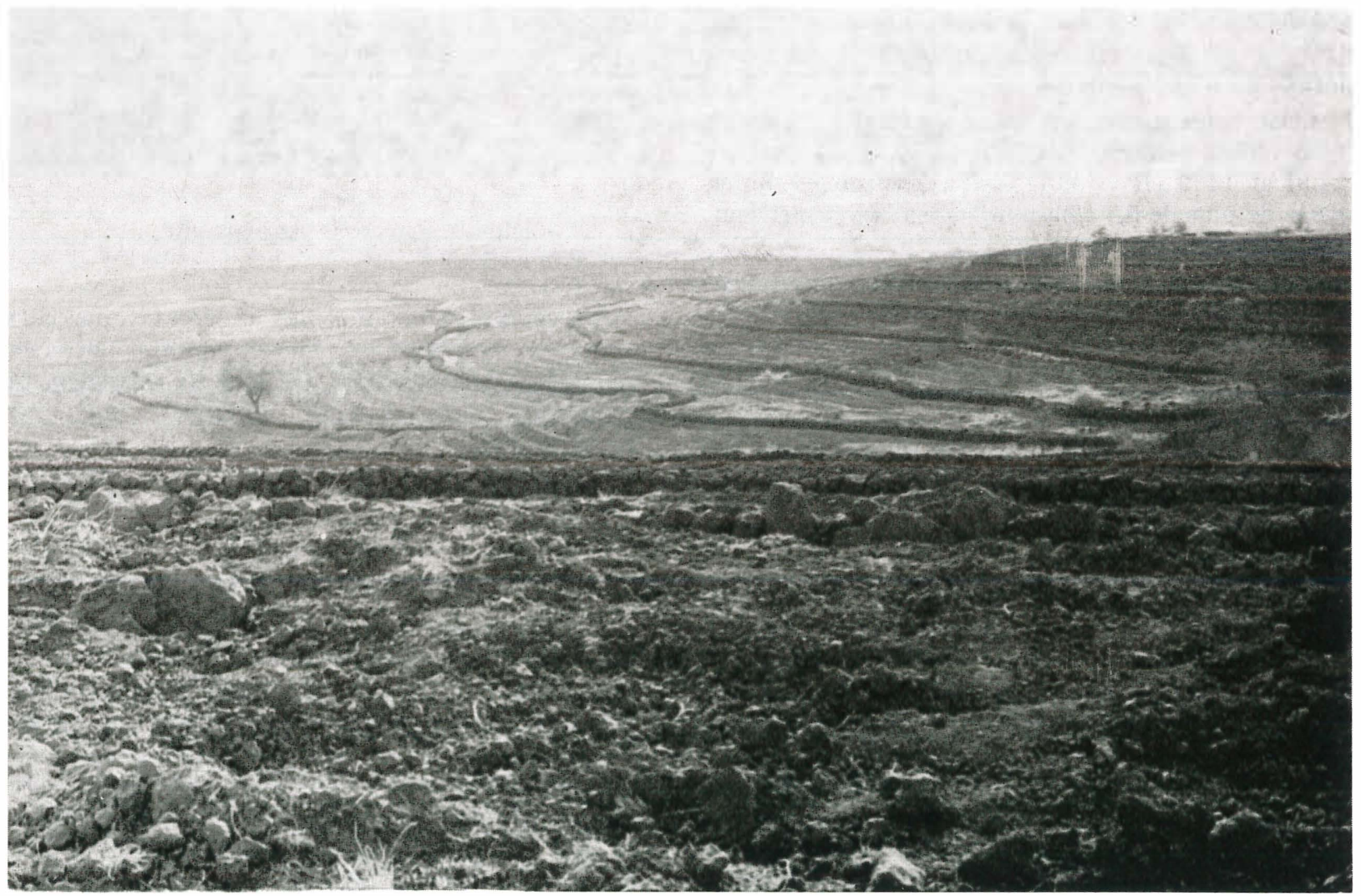


1969, Arachide : $P=506,8 \mathrm{~mm}$

\begin{tabular}{|c|c|c|}
\hline Parcelle & Poids kg/ha & Gain \% \\
\cline { 2 - 3 } & & \\
\cline { 2 - 3 } 1 & 1.502 & 89,4 \\
2 & 1.205 & 0 \\
3 & 1.282 & 51,9 \\
4 & & 61,6 \\
\hline
\end{tabular}

Quel que soit le dispositif antiérosif, le gain d'une parcelle traitée par rapport à la parcelle cultivée traditionnellement est très important et cette constatation se retrouve même lors des années les plus sèches où les rendements deviennent nuls sur la parcelle témoin.

$\mathrm{P} 1$ et $\mathrm{P} 4$ se révèlent plus intéressants que $\mathrm{P} 3$ comme traitement, P4 étant initialement meilleur que P1 mais perdant par la suite son avantage du fait de la dégradation progressive des ouvrages.

\section{2) TRAVAIL DU SOL.}

Les parcelles 1, 3 et 4 ont subi chaque année un labour réalisé en courbes de niveau.

Un billonnage a été effectué sur ces mêmes parcelles selon les courbes de niveau et ce billonnage a parfois été repris au cours de la saison.

Les binages effectués l'ont été systématiquement à la même date sur ces trois parcelles ainsi que sur la parcelle témoin lorsqu'ils se justifiaient sur celle-ci.

Le travail du sol modifie sensiblement les valeurs de l'érosion. a) Le rôle du labour et du billonnage est difficile à mettre en évidence du fait que chaque parcelle ainsi travaillée bénéficie en outre d'un traitement antiérosif. Cependant la parcelle 3 peut être considérée en début de saison des pluies comme étant sans traitement antiérosif, les lignes de végétation isohypses ne jouant alors que très imparfaitement leur rôle. La figure 11 qui fait la relation entre l'érosion et le facteur $R$ pour l'année 67, montre que la pente de la droite représentative de l'érosion est sensiblement moins forte pour P3 que pour P2.

Si nous considérons qu'avant le 24 juillet les lignes d'Andropogon ne jouent pas leur rôle, nous avons alors la comparaison des traitements suivañts :

P2 Parcelle témoin : érosion cumulée au 24 juillet, 5,5 t/ha ;

P3 Parcelle labourée et billonnée : érosion cumulée au 24 juillet, 2,0 t/ha ;

P1 Parcelle labourée et billonnée plus murets : érosion cumulée au 24 juillet, 0,6 t/ha.

Labour + billonnage réduisent donc l'érosion dans des proportions de l'ordre de 60 à $65 \%$. Le labour en courbes de niveau assorti, s'il se peut, de billonnage doit donc être un des grands thèmes de vulgarisation des services assurant l'animation rurale.

b) L'influence du binage est plus facile à mettre en évidence. Nous le verrons sur deux exemples :

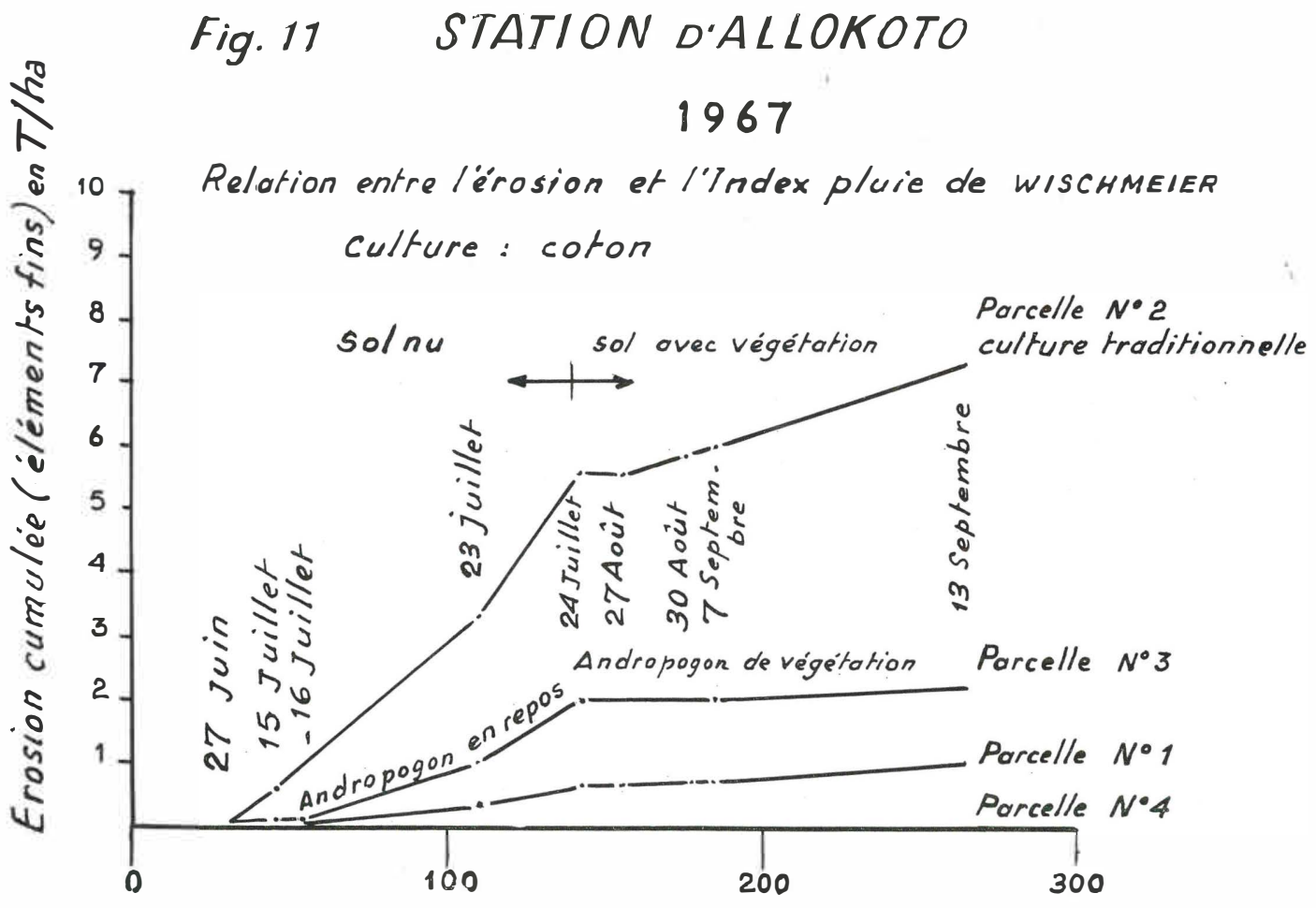




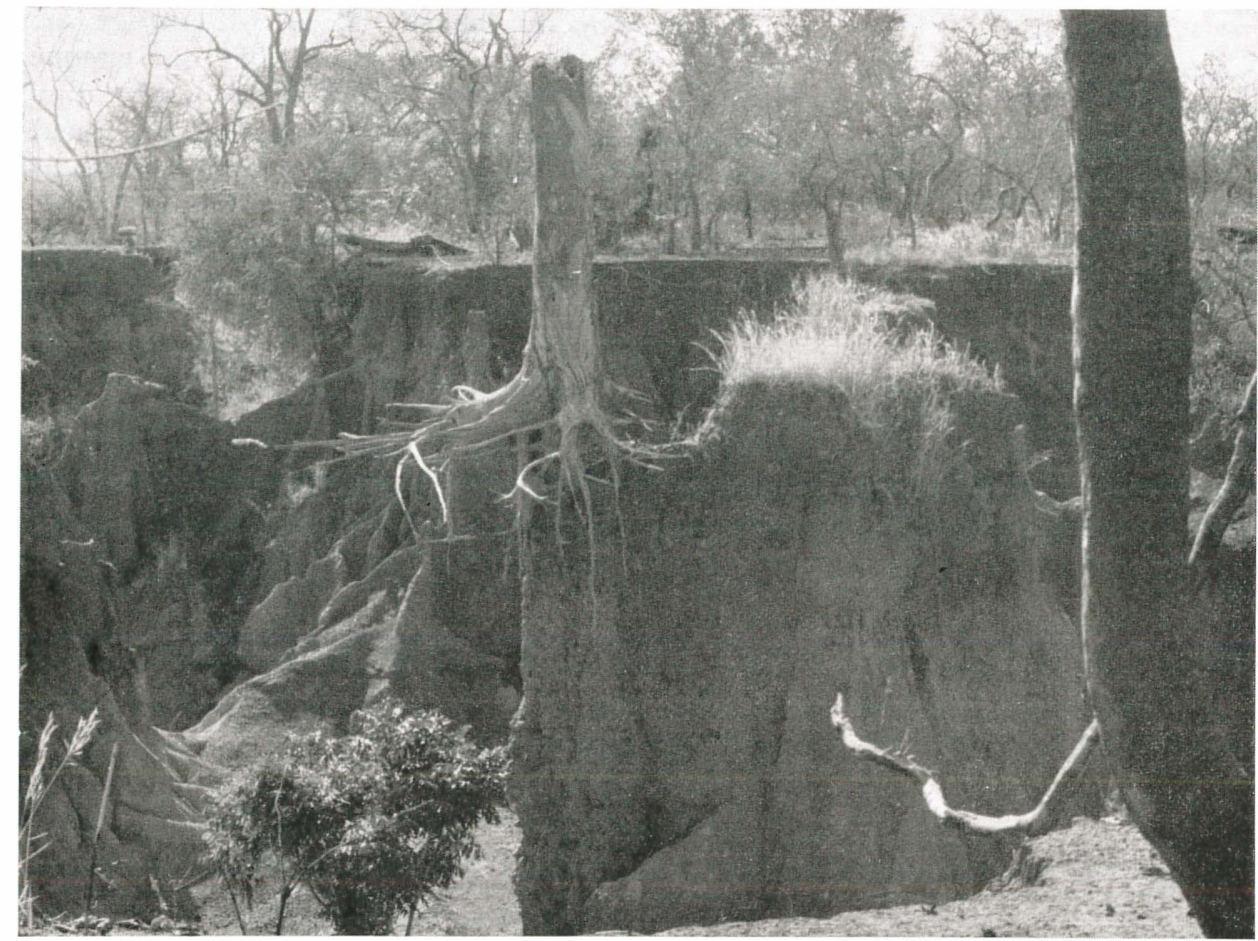

Parc national $d u$ " $W$ " au Niger. Erosion par écroulement de la falaise.

Photo Delwaulle.

la suivante. L'effet du binage est de rompre la croûte superficielle, ou "glaçage " qui se produit après une pluie et donc d'améliorer l'infiltration et le stockage de l'eau.

Limitant le ruissellement jusqu'à le rendre souvent nul, le binage limite du même fait les pertes en terre. Cependant, lorsqu'il y a malgré tout ruissellement, les concentrations en terre après binage sont plus fortes que sur sol glacé superficiellement.
- Prenons la parcelle $n^{0} 1$ en 1966. La tornade du 2 juin de $42,9 \mathrm{~mm}$ avec $I_{\max }$ de $132 \mathrm{~mm} / \mathrm{h}$ n'a donné que 5,25\% de ruissellement parce qu'elle venait derrière le labour. La tornade du 26 juin de $21,1 \mathrm{~mm}$ donnait lieu à un ruissellement de $24,9 \%$ : il n'y avait pas eu de binage depuis le 2 juin. Le 28 juin un binage était fait. Le 2 juillet survenait une tornade de $20,8 \mathrm{~mm}$ qui entraînait un ruissellement de $2,5 \%$. Le 3 juillet une deuxième tornade de $20,3 \mathrm{~mm}$ occasionnait un ruissellement de $29,1 \%$.

- En 1970, la pluie du 7 août intervient au milieu de l'opération binage. Les parcelles 1 et 2 sont binées, 3 et 4 ne le sont pas encore. La pluie de $12,3 \mathrm{~mm}$ n'occasionne aucun ruissellement sur 1 et 2 et respectivement $18,9 \%$ et $5,6 \%$ sur 3 et 4 : C'est le seul cas où la parcelle témoin P2 n'ayant pas ruisselé, d'autres parcelles l'ont fait.

On peut multiplier ce genre d'exemple. Celà tend donc à démontrer que l'idéal serait d'effectuer un binage après chaque tornade de façon à parer à

\section{3) RÔLE DE LA NATURE DU SOL.}

Les analyses et observations effectuées en 1966 avant l'implantation des parcelles avaient montré une variabilité plus importante à l'intérieur d'un champ entre partie haute et partie basse qu'entre les champs.

Nous avons essayé en 1967 de voir si à ces différences correspondaient des rendements distincts et le tableau 15 met en évidence un parallélisme étroit entre la teneur en eau à la capacité au champ*, le taux de matière organique totale d'une

* La capacité de rétention d'un sol est la quantité maximale d'eau capillaire retenue par le sol.

C'est une mesure théorique dont la valeur est souvent impossible à obtenir.

Il a été défìni une valeur pratique, la capacité au champ qui est la quantité d'eau retenue par le sol après un ressuyage de 48 heures après une pluie. Elle correspond à la capacité de rétention augmentée d'une grande partie de l'eau de gravité à écoulement lent.

TABLEAU 15

Allokoto, 1967, Relation sol-rendement des cultures

\begin{tabular}{|c|c|c|c|c|c|}
\hline & $\begin{array}{l}\text { Teneurs } \\
\text { en éléments } \\
\text { fins en } \%\end{array}$ & $\begin{array}{c}\text { Capacité } \\
\text { au champ \% }\end{array}$ & $\begin{array}{c}\text { Matière } \\
\text { organique } \\
\text { totale \% }\end{array}$ & $\begin{array}{l}\text { Rendement } \\
\text { coton graine } \\
\text { en } \mathrm{kg} / \mathrm{ha}\end{array}$ & $\begin{array}{c}\text { Hauteur } \\
\text { cotonniers } \\
\text { en cm }\end{array}$ \\
\hline Partie basse de la Parcelle 1 & 39 & 13,0 & 0,48 & 360 & 40 \\
\hline Partie haute de la Parcelle 1 & 60 & 18,3 & 1,08 & 980 & 90 \\
\hline
\end{tabular}


part, le rendement de coton graine en $\mathrm{kg} / \mathrm{ha}$, la hauteur moyenne des cotonniers, d'autre part.

- La teneur en eau à la capacité au champ a été calculée par l'analyse de prélèvements réalisés $24 \mathrm{~h}$ après une forte pluie $(63,5 \mathrm{~mm}$ du 13 septembre 67$)$.

- Le taux de matière organique est celui donné par les analyses initiales.

- Le rendement en coton graine donné en $\mathrm{kg} / \mathrm{ha}$ a été calculé par comptage des capsules avec pesage des capsules en fin de récolte. La hauteur des cotonniers a été mesurée en fìn de cycle (Défoliaison à $70 \%$ ).

Les différences constatées s'expliquent aisément par des variations dans la granulométrie des divers points du champ. Ces variations s'expliquent à leur tour par la position topographique de ces points : Dans le bas de la parcelle où les eaux de ruissellement ont eu le temps de prendre de la vitesse, les colloïdes, argiles et humus sont arrachés, contrairement aux points hauts de la parcelle où ce phénomène joue beaucoup moins. Le résultat est une baisse de la fertilité minérale et organique des parties basses accompagnée d'une diminution de la capacité de rétention en eau étant entendu que ces phénomènes se sont produits avant l'aménage- ment des parcelles. Ceci se traduit naturellement par une baisse importante du rendement des cotonniers.

Voici donc confirmés les méfaits de l'érosion :

- arrachage de la fraction colloïdale argile + humus, causant une diminution de la rétention en eau, diminution de la stabilité structurale, l'ensemble conduisant à un abaissement général de la fertilité.

Ces faits sont confirmés par les observations effectuées sur la parcelle $n^{\circ} 2$ où l'on peut mettre en évidence le rôle de l'eau sur la croissance des cotonniers ; dans les zones de départ de ruissellement la hauteur n'atteint que $20 \mathrm{~cm}$ avec une production nulle; autour des " chemins d'eau " la hauteur des cotonniers est de $80 \mathrm{~cm}$ avec des rendements allant de 600 à $700 \mathrm{~kg} / \mathrm{ha}$.

L'alimentation en eau des cotonniers est donc particulièrement importante, et les résultats très honorables de la parcelle $n^{\circ} 4$ montrent l'intérêt d'infiltrer la totalité de la pluviométrie.

\section{4) ÉTAT DE LA VÉGÉTATION.}

Au fur et à mesure que la saison avance la culture se développe et crée un frein à l'écoulement des

Photo Delwaulle.

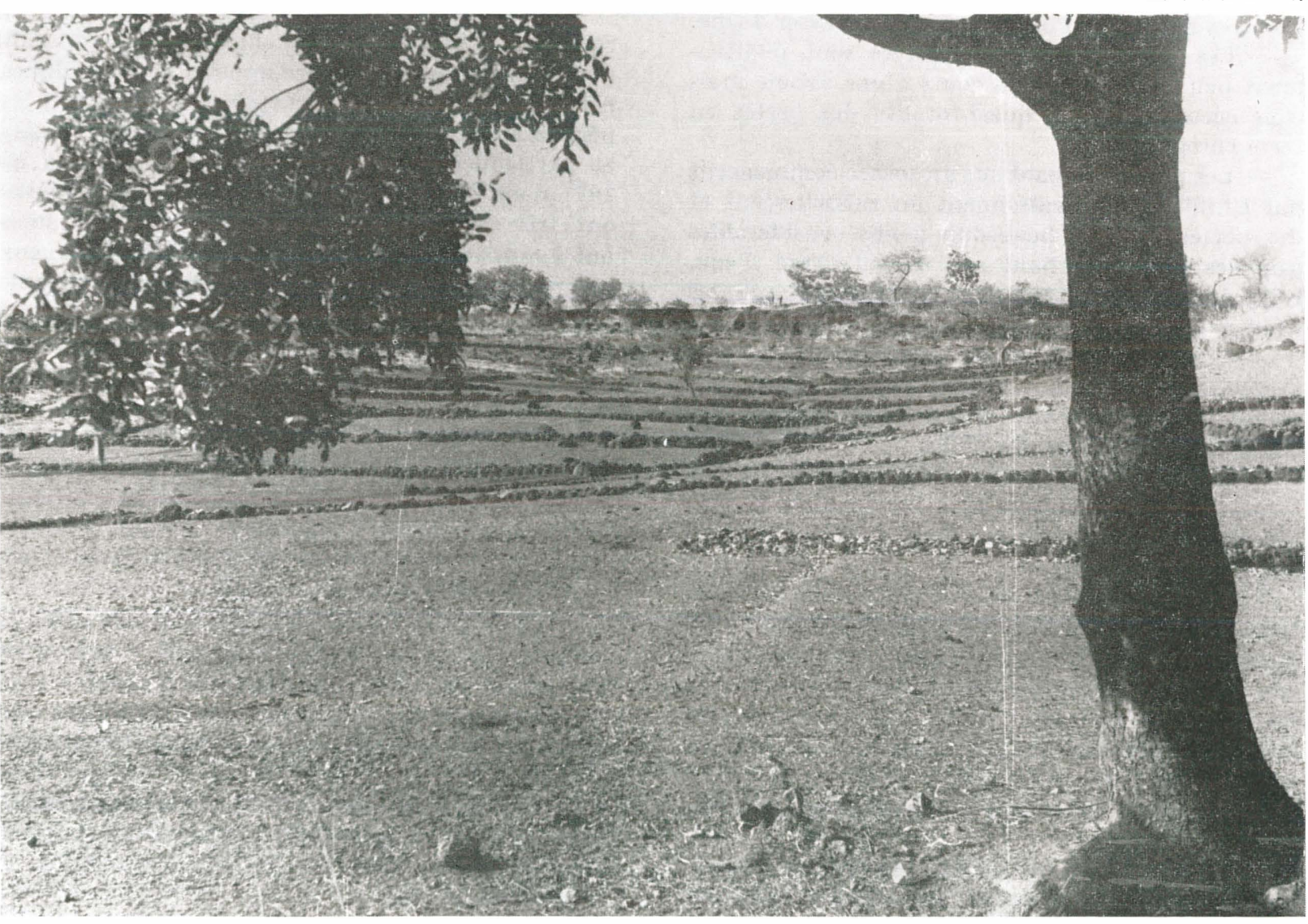


eaux. Ceci est mis en évidence si on essaie de faire le lien entre l'érosion et l'index $R$ et c'est ce que nous avons tenté de mettre en évidence sur la figure 11. Cette figure représente les pertes cumulées des terres exportées uniquement par les suspensions, en fonction des valeurs cumulées de l'index $R$, suivant les parcelles, pour la campagne 1967 .

Elle montre qu'il n'y a pas de relation directe entre l'érosion et $R$. Si une telle relation semble exister en début de saison des pluies, il se produit ensuite un ralentissement net de l'érosion en fonction de $R$ et ceci est dû au développement de la culture, en l'occurrence le coton.

Ceci est particulièrement net sur la parcelle 3 où l'efficacité du dispositif antiérosif est lié pour une part au développement de l'Andropogon. En début de saison des pluies, l'érosion y est beaucoup plus forte que sur les parcelles P1 et P4 mais cette érosion stoppe pratiquement avec le développement des lignes d'Andropogon à partir du 24 juillet.

\section{VIII. - CONCLUSION}

Les six ans d'observations sur l'érosion effectuées à Allokoto de 1966 à 1971 l'ont été pendant une période marquée par une pluviométrie inférieure à la moyenne. Les résultats doivent être examinés en tenant toujours compte que des années pluvieuses auront pour conséquence une érosion plus intense.

$\mathrm{Au}$ cours de ces six ans, nous avons pu mettre en évidence ou vérifier les aspects suivants de l'érosion :

- Pour déclencher des phénomènes d'érosion, la hauteur de la pluie doit être au moins de l'ordre de $12 \mathrm{~mm}$. Au-dessous, l'eau est infiltrée et ne donne lieu à aucune érosion.

- Le maximum de ruissellement est le fait de pluies de forte intensité. Les pertes en terre occasionnées par un seul orage peuvent dépasser $3 \mathrm{t} / \mathrm{ha}$.

- Ces pluies fortement érosives sont relativement peu nombreuses au cours d'une saison mais elles occasionnent la quasi-totalité des pertes en terre enregistrées.

- Les pluies tombant sur un sol sec commencent par s'infiltrer et occasionnent un ruissellement et des pertes en terre beaucoup moins considérables que des pluies tombant sur un sol gorgé d'eau. L'érosion est d'autant plus à craindre que les averses sont rapprochées.

- Les pluies de début de saison des pluies, plus groupées et avec un facteur $R$ relativement plus élevé occasionnent une érosion plus intense que celles de fin de saison des pluies.

- Les pourcentages de ruissellement sont très variables selon les pluies (0 à $70 \%$ ) mais le sont également d'une année sur l'autre ( 8,5 à $22,5 \%)$ sur parcelle témoin).

- L'érosion qui en est la conséquence est donc très variable d'une année à l'autre (3,5 t à $18,5 \mathrm{t} /$ ha pour la parcelle témoin). Il existe donc des années peu érosives et des années fortement érosives. Il est probable que les chiffres cités auraient été largement dépassés en cas d'année exceptionnellement pluvieuse mais ceux obtenus montrent bien l'extrême gravité des phénomènes d'érosion sous ces latitudes.

- Le travail du sol a pour conséquence de freiner l'érosion. A cet égard des binages fréquents réalisés après les pluies sont à préconiser. Le labour et le sillonnage selon les courbes de niveaux sont des façons culturales faciles à mettre en œuvre et qui ont un grand rôle dans la conservation des sols et de l'eau.

- Deux dispositifs antiérosifs ont donné entière satisfaction, murets et bourrelets armés aboutissant à la construction progressive de terrasses. Ces deux dispositifs sont donc à utiliser dès que le paysan a la possibilité de recueillir les pierres nécessaires sur son champ même.

- Les lignes de végétation isohypses ont une efficacité moindre mais leur action est suffisamment importante pour que cette méthode soit utilisée lorsque les pierres sont absentes.

- Ces trois types d'interventions sont simples, rustiques et se sont révélés efficaces. Il n'apparaît donc pas nécessaire de faire appel à des techniques plus évoluées ou plus luxueuses. A cet égard, les périmètres de DRS CES mis en place dans la Maggia et particulièrement dans la vallée d'Allokoto en 1971 dans le, cadre de l'aménagement de la région ont très certainement une grande efficacité, mais ont à notre sens l'inconvénient d'être trop luxueux et donc non réalisables par les paysans par l'exemple donné : Des réalisations plus modestes auraient peut-être déclenché une action en masse des paysans qui auraient pû être guidés par un service d'animation rurale.

- La formule de Ramser sur ce type de sol nous a donné satisfaction. Nous aurions tendance au vu des résultats de 66-71 à utiliser une formule moins sévère, mais il faut tenir compte d'années plus érosives que celles que nous avons rencontrées et nous continuerons donc à utiliser cette formule.

- Deux cultures : coton et arachide peu utilisées jusqu'à présent sur vertisols peuvent y donner de bons résultats. Elles peuvent donc s'inscrire dans un assolement en compagnie du sorgho. Les rendements des cultures sont fortement augmentés dès que le travail du sol (labour, billonnage) et un dispositif antiérosif permettent l'infiltration maximum des pluies et évitent les pertes en terre et singulièrement celles des éléments minéraux et organiques du sol. 